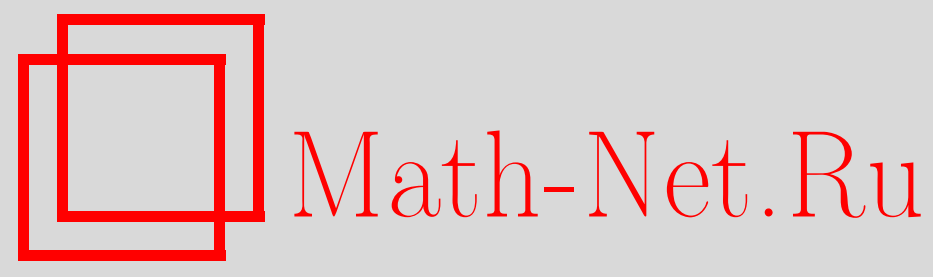

Б. Джеффрис, Г. В. Джонсон, Операторное исчисление Фейнмана для семейств некоммутирующих операторов: тензорные произведения, упорядоченные носители и выпутывание экспоненциального множителя, Матем. заметки, 2001, том 70, выпуск 6, 815-838

DOI: https://doi.org/10.4213/mzm795

Использование Общероссийского математического портала Math-Net.Ru подразумевает, что вы прочитали и согласны с пользовательским соглашением http://www . mathnet.ru/rus/agreement

Параметры загрузки:

IP : 3.81 .55 .215

26 апреля 2023 г., 13:52:30

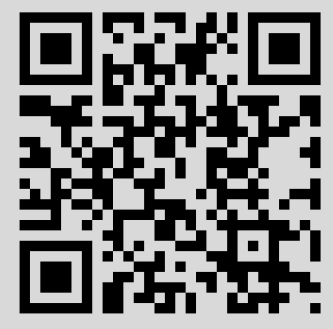




\title{
ОПЕРАТОРНОЕ ИСЧИСЛЕНИЕ ФЕЙНМАНА ДЛЯ СЕМЕЙСТВ НЕКОММУТИРУЮЩИХ ОПЕРАТОРОВ: ТЕНЗОРНЫЕ ПРОИЗВЕДЕНИЯ, УПОРЯДОЧЕННЫЕ НОСИТЕЛИ И ВЫПУТЫВАНИЕ ЭКСПОНЕНЦИАЛЬНОГО МНОЖИТЕЛЯ
}

\author{
Б. Джеффрис, Г. В. Джонсон
}

\begin{abstract}
В недавно появившейся работе авторы изложили основные идеи, используемые в их подходе к фейнмановскому операторному исчислению систем некоммутирующих линейных ограниченных операторов, действуюших в банаховом пространстве. Центральньм объектом этой теории является вьпутывающая алгебра (коммутативная банахова алгебра) и выпутывающее отображение коммутативной структуры в алгебру некоммутирующих операторов. Цель настоящей статьи состоит в изучении свойств выпутывающего отображения с акцентом на (i) формулу Фейнмана для выпутывания экспоненциального множителя и (ii) зависимость вьпутьвающего отображения от упорядоченных носителей мер, управляющих выпутыванием.
\end{abstract}

Библиография: 13 названий.

Введение. Для ряда разделов математики и приложений важно иметь возможность вычислять функции от операторов. При наличии единственного самосопряженного оператора или нескольких коммутируюших операторов спектральная теорема обеспечивает чрезвычайно богатые возможности для функционального исчисления. Однако, как только мы имеем два или более некоммутирующих оператора, функциональное исчисление становится значительно более сложным даже для самосопряженных операторов. Однозначность упорядочения операторов является одним из принципиальных обстоятельств, с которыми приходится иметь дело.

Операторное исчисление Фейнмана [1] возникло как следствие интереса к эволюционируюшим квантовым системам и, в частности, нашло отражение в его знаменитой работе по квантовой электродинамике [2]. Операторное исчисление Фейнмана возникло также под влиянием его ранней работы по функциональному интегрированию [3]; в действительности он рассматривал операторное исчисление как некоторое обобщение функционального интегрирования.

Мы начнем с очень краткого изложение идей Фейнмана о конструкции функций от некоммутирующих операторов. Более подробное рассмотрение эвристических идей $\Phi$ ейнмана содержится в [4], значительно больше деталей читатель может найти в [5, гл. 14].

Индексы времени используются для того, чтобы установить порядок действия операций в произведениях. Операторы с меньшими индексами времени действуют раньше 
операторов с большими индексами независимо от того, как они стоят в формулах. Функции от операторов, снабженных временным индексом, строятся так же, как и в случае коммутируюших операторов. Соотношения, полученные после такой процедуры, обычно оказьваются неправильными, если их интерпретировать обычным образом. Наконец, операторные выражения могут быть приведены к естественному порядку, иначе говоря, "распутаны". Последний шаг обычно оказывается трудным. Грубо говоря, этот шаг состоит в последовательности преобразований операторных выражений до тех пор, пока порядок операций в формулах не будет соответствовать временному упорядочению.

В силу своего особого интереса к эволюционирующим квантовым системам Фейнман интересовался экспонентами сумм некоммутирующих операторов. Во многих случаях один из операторов часто играет доминирующую роль, а остальные операторы рассматриваются как его возмущения. Поэтому важно иметь формулу выпутьвания, учитывающую роль доминирующего оператора. Основной результат статьи Фейнмана [1] решает эту задачу. Фейнман назьвает это соотношение “формулой вьпутьвания экспериментального множителя". Мы заменяем слово “экспериментальньй” словом “экспоненциальньй", поскольку это лучше соответствует нашему математическому подходу. Основной результат этой статьи - математически строгая формулировка и доказательство формулы Фейнмана и некоторых аналогичных соотношений (см. п. 5).

Читатель мог заметить слово “исчисление" в названии статьи. Каждому оператору будет сопоставлена некоторая мера. Эти меры определяют некоторое специальное операторное исчисление, которое изменится, если изменить набор мер. В этой статье мы изучим случай, когда носители мер упорядочены (или частично упорядочены). Если носители упорядочены, то теория, развитие которой началось в [4], рассматривается нами в связи с элементарными аспектами работ Маслова [6] и Назайкинского, Стернина и Шаталова [7].

Дальнейшие результаты этой работы используют прямые суммы банаховых пространств, условие симметричности выпутывающего отображения, ряды, тензорные произведения и меры, отличные от вероятностньх. Все эти результаты весьма элементарны, однако они представляют потенциальный интерес. Например, хотя вероятностные меры и являются естественньпи объектами, связанньпи с нашим функциональным исчислением, такой выбор не всегда является наилучшим. Меры на $[0, t]$, масса которых растет с увеличением $t$, являются естественными для эволюционных задач [8]. Далее, даже в том случае, когда используется вероятностная мера, иногда оказьвается полезным сузить меру на подмножество, где она, конечно, может не быть вероятностной мерой.

Меры, сопоставляемые связанньм с ними операторам, играют важную роль в нашей теории. Например, можно изучать и сравнивать между собой различные операторные исчисления в рамках единого подхода. Далее, теоремы устойчивости мер типа [9] могут быть использованы для ответа на естественные вопросы, а также для того, чтобы помочь понять конкретное операторное исчисление, рассматривая его как предел более простого операторного исчисления (см. например, [10]). Дополнительные комментарии о роли мер, а также ссылки на более ранние работы на эту тему можно найти во введении к статье [4].

Сравнивая эту статью и ближайшие предшествующие работы [4], [9]-[11] с работой Фейнмана [1], можно заметить, что мы расширяем и интерпретируем идеи Фейнмана, а 
также придаем им математическую строгость. В действительности, сходное утверждение справедливо для большей части математически строгих работ на эту тему (ссылки см. в [4] и $[5])$.

Основные определения и факты из [4] будут кратко рассмотрены в п. 1. Более детальное изложение содержится в разделах 1 и 2 статьи [4].

1. Банаховы алгебры $\mathbb{A}$ и $\mathbb{D}$ и выпутывающие отображения. Мы начнем с обзора свойств двух коммутативньх банаховых алгебр $\mathbb{A}$ и $\mathbb{D}$ и отметим тесную связь между ними.

Для заданного целого положительного $n$ и положительных чисел $r_{1}, \ldots, r_{n}$ обозначим через $\mathbb{A}\left(r_{1}, \ldots, r_{n}\right)$, или кратко $\mathbb{A}$, пространство комплекснозначных функций $\left(z_{1}\right.$, $\left.\ldots, z_{n}\right) \mapsto f\left(z_{1}, \ldots, z_{n}\right)$ от $n$ комплексных переменных, аналитических в точке $(0, \ldots, 0)$ и таких, что их разложение в степенной ряд

$$
f\left(z_{1}, \ldots, z_{n}\right)=\sum_{m_{1}, \ldots, m_{n}=0}^{\infty} c_{m_{1}, \ldots, m_{n}} z_{1}^{m_{1}} \cdots z_{n}^{m_{n}}
$$

сходится абсолютно по крайней мере на замкнутом полидиске $\left|z_{1}\right| \leqslant r_{1}, \ldots,\left|z_{n}\right| \leqslant r_{n}$. Такие функции являются аналитическими по крайней мере на открытом полидиске $\left|z_{1}\right|<r_{1}, \ldots,\left|z_{n}\right|<r_{n}$. Заметим, что любая целая функция $n$ комплексных переменных принадлежит пространству $\mathbb{A}\left(r_{1}, \ldots, r_{n}\right)$ для всех положительных чисел $r_{1}, \ldots, r_{n}$.

Для функций $f \in \mathbb{A}\left(r_{1}, \ldots, r_{n}\right)$, заданных соотношением (1.1), мы положим

$$
\|f\|=\|f\|_{\mathbb{A}\left(r_{1}, \ldots, r_{n}\right)}=\sum_{m_{1}, \ldots, m_{n}=0}^{\infty}\left|c_{m_{1}, \ldots, m_{n}}\right| r_{1}^{m_{1}} \cdots r_{n}^{m_{n}}
$$

Функция на $\mathbb{A}\left(r_{1}, \ldots, r_{n}\right)$, определенная формулой $(1.1)$, отображает $\mathbb{A}\left(r_{1}, \ldots, r_{n}\right)$ в коммутативную банахову алгебру относительно поточечных операций.

Рассмотрим банахову алгебру $\mathbb{D}$. Пусть $X$ - банахово пространство, и пусть $A_{1}, \ldots$, $A_{n}$ - ненулевые операторы из пространства линейных ограниченных операторов $\mathscr{L}(X)$ над $X$. За исключением чисел $\left\|A_{1}\right\|, \ldots,\left\|A_{n}\right\|$, которые будут использоваться как веса, мы игнорируем специфические свойства $A_{1}, \ldots, A_{n}$ как операторов и вводим коммутативную банахову алгебру, состоящую из "аналитических функций" $f\left(\widetilde{A}_{1}, \ldots, \widetilde{A}_{n}\right)$, где $\widetilde{A}_{1}, \ldots, \widetilde{A}_{n}$ интерпретируются как произвольные коммутирующие объекты.

Рассмотрим множество $\mathbb{D}=\mathbb{D}\left(A_{1}, \ldots, A_{n}\right)$ всех выражений вида

$$
f\left(\widetilde{A}_{1}, \ldots, \widetilde{A}_{n}\right)=\sum_{m_{1}, \ldots, m_{n}=0}^{\infty} c_{m_{1}, \ldots, m_{n}} \widetilde{A}_{1}^{m_{1}} \ldots \widetilde{A}_{n}^{m_{n}}
$$

где $c_{m_{1}, \ldots, m_{n}} \in \mathbb{C}$ для всех $m_{1}, \ldots, m_{n}=0,1, \ldots$ и

$$
\begin{aligned}
\left\|f\left(\widetilde{A}_{1}, \ldots, \widetilde{A}_{n}\right)\right\| & =\left\|f\left(\widetilde{A}_{1}, \ldots, \widetilde{A}_{n}\right)\right\|_{\mathbb{D}\left(A_{1}, \ldots, A_{n}\right)} \\
& =\sum_{m_{1}, \ldots, m_{n}=0}^{\infty}\left|c_{m_{1}, \ldots, m_{n}}\right|\left\|A_{1}\right\|^{m_{1}} \ldots\left\|A_{n}\right\|^{m_{n}}<\infty .
\end{aligned}
$$


$\Phi$ ункция на $\mathbb{D}\left(A_{1}, \ldots, A_{n}\right)$, заданная формулой $(1.3)$, отображает $\mathbb{D}\left(A_{1}, \ldots, A_{n}\right)$ в коммутативную банахову алгебру относительно поточечных операций. Действительно, если мы возьмем $r_{j}=\left\|A_{j}\right\|$ для $j=1, \ldots, n$, то $\mathbb{D}\left(A_{1}, \ldots, A_{n}\right)$ просто получается из $\mathbb{A}\left(r_{1}, \ldots, r_{n}\right)$ при переобозначении переменных. Поэтому $\mathbb{D}$ и $\mathbb{A}$ естественным образом изометрически изоморфны как банаховы алгебры.

Мы назьваем $\mathbb{D}\left(A_{1}, \ldots, A_{n}\right)$ вылутыв вююей алгеброй, связанной с набором $\left(A_{1}, \ldots\right.$, $A_{n}$ ) ограниченных линеных операторов, действующих в $X$.

Пусть набор $A_{1}, \ldots, A_{n}$ состоит из ненулевых операторов из $\mathscr{L}(X)$, и пусть $\mu_{1}, \ldots, \mu_{n}$ - непрерьвные вероятностные меры, заданные на множестве $\mathscr{B}[0,1]$ борелевских подмножеств отрезка $[0,1]$. Непрерьвность означает, что мера любого одноточечного множества равна нулю.

Идея, которой мы следуем, состоит в замене операторов $A_{1}, \ldots, A_{n}$ элементами $\widetilde{A}_{1}$, $\ldots, \widetilde{A}_{n}$ из $\mathbb{D}$, затем мы конструируем требуемую функцию переменных $\widetilde{A}_{1}, \ldots, \widetilde{A}_{n}$. Пока мы работаем в алгебре $\mathbb{D}$, мы упорядочиваем выражение для функции и затем переходим обратно в алгебру $\mathscr{L}(X)$, просто снимая знаки тильда. Теперь мы продемонстрируем формально только что сформулированную идею.

Для заданных неотрицательных $m_{1}, \ldots, m_{n}$ положим

$$
P^{m_{1}, \ldots, m_{n}}\left(z_{1}, \ldots, z_{n}\right)=z_{1}^{m_{1}} \cdots z_{n}^{m_{n}}
$$

так что

$$
P^{m_{1}, \ldots, m_{n}}\left(\widetilde{A}_{1}, \ldots, \widetilde{A}_{n}\right)=\widetilde{A}_{1}^{m_{1}} \ldots \widetilde{A}_{n}^{m_{n}} .
$$

Для каждого $m=0,1, \ldots$ через $S_{m}$ обозначим множество всех перестановок целых чисел $\{1, \ldots, m\}$, и для заданной перестановки $\pi \in S_{m}$ пусть

$$
\Delta_{m}(\pi)=\left\{\left(s_{1}, \ldots, s_{m}\right) \in[0,1]^{m}: 0<s_{\pi(1)}<\cdots<s_{\pi(m)}<1\right\} .
$$

Для $j=1, \ldots, n$ и всех $s \in[0,1]$ положим

$$
\widetilde{A}_{j}(s)=\widetilde{A}_{j}
$$

Теперь для неотрицательных целых чисел $m_{1}, \ldots, m_{n}$ и $m=m_{1}+\cdots+m_{n}$ положим по определению

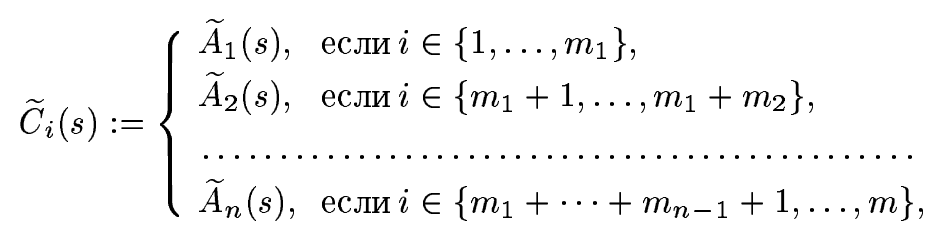

для $i=1, \ldots, m$ и $0 \leqslant s \leqslant 1$. Хотя $\widetilde{C}_{i}(s)$ зависит от неотрицательных целых $m_{1}, \ldots, m_{n}$, в обозначениях для упрошения записи следующих формул мы игнорируем эту зависимость.

ПРЕДЛОЖЕНИЕ 1.1. Имеет место соотношение

$$
\begin{aligned}
& P^{m_{1}, \ldots, m_{n}}\left(\widetilde{A}_{1}, \ldots, \widetilde{A}_{n}\right) \\
& \quad=\sum_{\pi \in S_{m}} \int_{\Delta_{m}(\pi)} \widetilde{C}_{\pi(m)}\left(s_{\pi(m)}\right) \cdots \widetilde{C}_{\pi(1)}\left(s_{\pi(1)}\right)\left(\mu_{1}^{m_{1}} \times \cdots \times \mu_{n}^{m_{n}}\right)\left(d s_{1}, \ldots, d s_{m}\right) .
\end{aligned}
$$


ДоКАЗАТЕЛЬСТвО приводится в $[4$, разд. 2].

Теперь мы можем определить отображение $\mathscr{T}_{\mu_{1}, \ldots, \mu_{n}}$, которое вернет нас от коммутативного случая к некоммутативной ситуации для $\mathscr{L}(X)$. Нам необходимы обозначения такие же, как и в $(1.8)$ и $(1.9)$, но использущие $A_{1}, \ldots, A_{n}$ вместо $\widetilde{A}_{1}, \ldots, \widetilde{A}_{n}$. Соответственно для $j=1, \ldots, n$ и всех $s \in[0,1]$ мы полагаем

$$
A_{j}(s)=A_{j},
$$

а для $i=1, \ldots, m$ положим

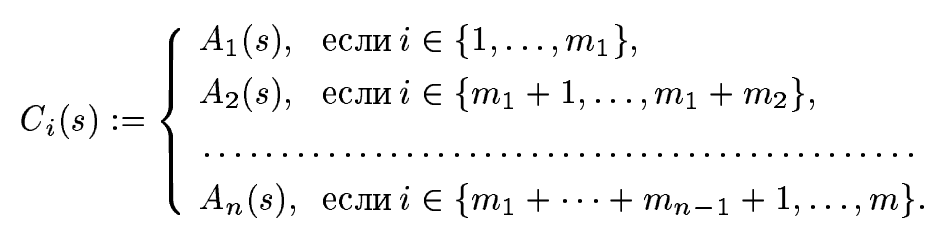

ОПРЕДЕЛЕНИЕ 1.2. Отображение $\mathscr{T}_{\mu_{1}, \ldots, \mu_{n}}$ задается следующим образом:

$$
\begin{aligned}
\mathscr{T}_{\mu_{1}, \ldots, \mu_{n}}\left(P^{m_{1}, \ldots, m_{n}}\left(\widetilde{A}_{1}, \ldots, \widetilde{A}_{n}\right)\right)= & \sum_{\pi \in S_{m}} \int_{\Delta_{m}(\pi)} C_{\pi(m)}\left(s_{\pi(m)}\right) \cdots C_{\pi(1)}\left(s_{\pi(1)}\right) \\
& \times\left(\mu_{1}^{m_{1}} \times \cdots \times \mu_{n}^{m_{n}}\right)\left(d s_{1}, \ldots, d s_{n}\right) .
\end{aligned}
$$

Далее, для функции $f\left(\widetilde{A}_{1}, \ldots, \widetilde{A}_{n}\right) \in \mathbb{D}\left(A_{1}, \ldots, A_{n}\right)$, заданной соотношением

$$
f\left(\widetilde{A}_{1}, \ldots, \widetilde{A}_{n}\right)=\sum_{m_{1}, \ldots, m_{n}=0}^{\infty} c_{m_{1}, \ldots, m_{n}} \widetilde{A}_{1}^{m_{1}} \ldots \widetilde{A}_{n}^{m_{n}},
$$

мы полагаем $\mathscr{T}_{\mu_{1}, \ldots, \mu_{n}}\left(f\left(\widetilde{A}_{1}, \ldots, \widetilde{A}_{n}\right)\right)$ равным

$$
\sum_{m_{1}, \ldots, m_{n}=0}^{\infty} c_{m_{1}, \ldots, m_{n}} \mathscr{T}_{\mu_{1}, \ldots, \mu_{n}}\left(P^{m_{1}, \ldots, m_{n}}\left(\widetilde{A}_{1}, \ldots, \widetilde{A}_{n}\right)\right) .
$$

Как обычно, мы будем писать оператор $\mathscr{T}_{\mu_{1}, \ldots, \mu_{n}} x$ вместо $\mathscr{T}_{\mu_{1}, \ldots, \mu_{n}}(x)$ для элемента $x$ из $\mathbb{D}\left(A_{1}, \ldots, A_{n}\right)$. Следующее предложение дает нам основные свойства вьпутывающего отображения; доказательство можно найти в [4, разд. 2].

ПРЕДЛОЖЕНИЕ 1.3. (i) Ряд (1.15) сходится абсолютно в равномерной операторной топологии $\mathscr{L}(X)$ для всех $f\left(\widetilde{A}_{1}, \ldots, \widetilde{A}_{n}\right) \in \mathbb{D}\left(A_{1}, \ldots, A_{n}\right)$.

(ii) $\mathscr{T}_{\mu_{1}, \ldots, \mu_{n}}$ является линейным отображением из $\mathbb{D}\left(A_{1}, \ldots, A_{n}\right)$ в $\mathscr{L}(X)$.

(iii) Для всех $f\left(\widetilde{A}_{1}, \ldots, \widetilde{A}_{n}\right) \in \mathbb{D}\left(A_{1}, \ldots, A_{n}\right)$ имеем

$$
\left\|\mathscr{T}_{\mu_{1}, \ldots, \mu_{n}} f\left(\widetilde{A}_{1}, \ldots, \widetilde{A}_{n}\right)\right\|_{\mathscr{L}(X)} \leqslant\left\|f\left(\widetilde{A}_{1}, \ldots, \widetilde{A}_{n}\right)\right\|_{\mathbb{D}\left(A_{1}, \ldots, A_{n}\right)} .
$$

Действительно,

$$
\left\|\mathscr{T}_{\mu_{1}, \ldots, \mu_{n}}\right\|=1
$$


Для краткости и чтобы выделить тот факт, что $\mathscr{T}_{\mu_{1}, \ldots, \mu_{n}} f\left(\widetilde{A}_{1}, \ldots, \widetilde{A}_{n}\right)$ следует понимать как функцию от необязательно коммутирующих операторов $A_{1}, \ldots, A_{n}$, мы часто используем альтернативное обозначение, приводимое в следующих двух равенствах:

$$
\begin{aligned}
f_{\mu_{1}, \ldots, \mu_{n}}\left(A_{1}, \ldots, A_{n}\right) & :=\mathscr{T}_{\mu_{1}, \ldots, \mu_{n}} f\left(\widetilde{A}_{1}, \ldots, \widetilde{A}_{n}\right), \\
P_{\mu_{1}, \ldots, \mu_{n}}^{m_{1}, \ldots, m_{n}}\left(A_{1}, \ldots, A_{n}\right) & :=\mathscr{T}_{\mu_{1}, \ldots, \mu_{n}} P^{m_{1}, \ldots, m_{n}}\left(\widetilde{A}_{1}, \ldots, \widetilde{A}_{n}\right) .
\end{aligned}
$$

Формулы (1.13) и (1.15) во многих случаях являются полезными, но они имеют одно неприятное свойство. Мы ограничимся замечаниями по поводу (1.13), но они также имеют силу и по отношению к (1.15). В формуле (1.13) члены повторяются многократно. Это обстоятельство желательно устранить не только по чисто математическим причинам, но также целесообразно с точки зрения приложений.

Сначала мы объясним, откуда появляется альтернативная версия формулы (1.13), и тогда сформулируем результат. Пусть дано $\pi \in S_{m}$. Поскольку $A_{1}$ повторяется $m_{1}$ раз, $A_{2}-m_{2}$ раз, .., и $A_{n}-m_{n}$ раз, то полное число перестановок, приводящее к тому же выражению, равно $m_{1} ! m_{2} ! \cdots m_{n} !$. Проверяя формулу $(1.12)$, мы видим, что эти перестановки состоят из перестановок, переставляющих индексы $s_{1}, \ldots, s_{m_{1}}$ между собой, переставляющих индексы $s_{m_{1}+1}, \ldots, s_{m_{1}+m_{2}}$ между собой, $\ldots$, и, наконец, переставляюших индексы $s_{m_{1}}+\cdots+m_{n-1}+1, \ldots, s_{m}$ между собой. Мы можем сократить нашу формулу, выбирая лишь одну из перестановок, умноженную на $m_{1} ! m_{2} ! \cdots m_{n} !$, поскольку для каждого $j=1, \ldots, n$ мера $\mu_{j}^{m_{j}}$ левоинвариантна относительно перестановок переменных $s_{m_{1}}+\cdots+m_{j-1}+1, \ldots, s_{m_{1}}+\cdots+m_{j-1}+m_{j}$. Удобно выбрать перестановку, для которой

$$
s_{1}<\cdots<s_{m_{1}}, \quad s_{m_{1}+1}<\cdots<s_{m_{1}+m_{2}}, \quad \cdots, \quad s_{m_{1}+\cdots+m_{n-1}+1}<\cdots<s_{m} .
$$

Таким образом, мы получаем

$$
\begin{aligned}
& \mathscr{T}_{\mu_{1}, \ldots, \mu_{n}} P^{m_{1}, \ldots, m_{n}}\left(\widetilde{A}_{1}, \ldots, \widetilde{A}_{n}\right) \\
& =m_{1} ! \cdots m_{n} ! \sum_{\pi \in \mathscr{P}_{m_{1}, \ldots, m_{n}}} \int_{\left(\Delta_{m_{1}} \times \cdots \times \Delta_{m_{n}}\right)(\pi)} C_{\pi(m)}\left(s_{\pi(m)}\right) \times \cdots \times C_{\pi(1)}\left(s_{\pi(1)}\right) \\
& \quad \times\left(\mu_{1}^{m_{1}} \times \cdots \times \mu_{n}^{m_{n}}\right)\left(d s_{1}, \ldots, d s_{m}\right),
\end{aligned}
$$

где

$$
\begin{aligned}
& \Delta_{m_{1}}=\left\{\left(s_{1}, \ldots, s_{m_{1}}\right) \in[0,1]^{m}: 0<s_{1}<\cdots<s_{m_{1}}<1\right\} \\
& \Delta_{m_{n}}=\left\{\left(s_{m_{1}+\cdots+m_{n-1}+1}, \ldots, s_{m}\right) \in[0,1]^{m_{n}}: 0<s_{m_{1}+\cdots+m_{n-1}+1}<\cdots<s_{m}<1\right\}
\end{aligned}
$$

И

$$
\left(\Delta_{m_{1}} \times \cdots \times \Delta_{m_{n}}\right)(\pi)=\left\{\left(s_{1}, \ldots, s_{m}\right) \in \Delta_{m_{1}} \times \cdots \times \Delta_{m_{n}}: 0<s_{\pi(1)}<\cdots<s_{\pi(m)}<1\right\},
$$

а $\mathscr{P}_{m_{1}, \ldots, m_{n}}-$ множество перестановок $\pi \in S_{m}$, которые оставляют инвариантным относительньй порядок точек $s_{1}, \ldots, s_{m_{1}}$, а также оставляют инвариантным относительный порядок точек $s_{m_{1}+1}, \ldots, s_{m_{1}+m_{2}}$ и, наконец, оставляют инвариантньм относительный порядок точек $s_{m_{1}}+\cdots+m_{n-1}+1, \ldots, s_{m}$. Мощность множества $\mathscr{P}_{m_{1}, \ldots, m_{n}}$ вычисляется следующим образом:

$$
\operatorname{Card}\left(\mathscr{P}_{m_{1}, \ldots, m_{n}}\right)=\frac{\left(m_{1}+\cdots+m_{n}\right) !}{m_{1} ! \cdots m_{n} !}=\frac{m !}{m_{1} ! \cdots m_{n} !} .
$$

Теперь мы сформулируем предложение, которое выше мы уже доказали. 
ПРЕДЛОЖЕНИЕ 1.4. Onератор

$$
P_{\mu_{1}, \ldots, \mu_{n}}^{m_{1}, \ldots, m_{n}}\left(A_{1}, \ldots, A_{n}\right)=\mathscr{T}_{\mu_{1}, \ldots, \mu_{n}} P^{m_{1}, \ldots, m_{n}}\left(\widetilde{A}_{1}, \ldots, \widetilde{A}_{n}\right),
$$

заданный формулой (1.13), имеет представление (1.20); обозначения оббясняются в (1.21), (1.22) и в приведенном выше описании множества перестановок $\mathscr{P}_{m_{1}, \ldots, m_{n}}$.

2. Прямые суммы, симметрии, ряды и тензорные произведения. Начнем с рассмотрения случая, когда банахово пространство $X$ является прямой суммой банаховых пространств $Y$ и $Z$ и каждый оператор $A_{j}$ является соответствующей прямой суммой операторов.

ПРЕДЛОЖЕНИЕ 2.1. Предположим, что $X=Y \oplus Z$ и существуют операторь $B_{j} \in \mathscr{L}(Y)$ и $C_{j} \in \mathscr{L}(Z)$ такие, что $A_{j}=B_{j} \oplus C_{j}$ для кажсдого $j=1, \ldots, n$. Тогда

$$
P_{\mu_{1}, \ldots, \mu_{n}}^{m_{1}, \ldots, m_{n}}\left(A_{1}, \ldots, A_{n}\right)=P_{\mu_{1}, \ldots, \mu_{n}}^{m_{1}, \ldots, m_{n}}\left(B_{1}, \ldots, B_{n}\right) \oplus P_{\mu_{1}, \ldots, \mu_{n}}^{m_{1}, \ldots, m_{n}}\left(C_{1}, \ldots, C_{n}\right) .
$$

Аналогично, если функиия $f \in \mathbb{A}\left(\left\|A_{1}\right\|,\left\|A_{2}\right\|, \ldots,\left\|A_{n}\right\|\right)$ задана формулой (1.1), то $f$ принадлежит $\mathbb{A}\left(\left\|B_{1}\right\|, \ldots,\left\|B_{n}\right\|\right) \cap \mathbb{A}\left(\left\|C_{1}\right\|, \ldots,\left\|C_{n}\right\|\right)$ u

$$
f_{\mu_{1}, \ldots, \mu_{n}}\left(A_{1}, \ldots, A_{n}\right)=f_{\mu_{1}, \ldots, \mu_{n}}\left(B_{1}, \ldots, B_{n}\right) \oplus f_{\mu_{1}, \ldots, \mu_{n}}\left(C_{1}, \ldots, C_{n}\right) .
$$

ДокАЗАТЕЛЬСТво. Пусть $Q$ - проектор, коммутирующий с каждым из операторов $A_{1}, \ldots, A_{n}$. Проекциями, важньми здесь для нас, являются проекции из $X$ на $Y$ и $Z$ соответственно. Из определения 1.2 и того факта, что $Q^{n}=Q$, получаем

$$
P_{\mu_{1}, \ldots, \mu_{n}}^{m_{1}, \ldots, m_{n}}\left(A_{1} Q, \ldots, A_{n} Q\right)=P_{\mu_{1}, \ldots, \mu_{n}, \mu_{n}}^{m_{1}, \ldots, m_{n}, 1}\left(A_{1}, \ldots, A_{n}, Q^{n}\right) .
$$

Но предложение 3.3 из [4] утверждает, что поскольку $Q$ коммутирует со всеми операторами $A_{1}, \ldots, A_{n}, Q$ может быть исключено из вьпутьвающей формулы; в частности,

$$
P_{\mu_{1}, \ldots, \mu_{n}, \mu_{n}}^{m_{1}, \ldots, m_{n}, 1}\left(A_{1}, \ldots, A_{n}, Q\right)=Q P_{\mu_{1}, \ldots, \mu_{n}}^{m_{1}, \ldots, m_{n}}\left(A_{1}, \ldots, A_{n}\right) .
$$

Комбинируя последнее равенство с двумя предыдущими, мы получаем

$$
P_{\mu_{1}, \ldots, \mu_{n}}^{m_{1}, \ldots, m_{n}}\left(A_{1} Q, \ldots, A_{n} Q\right)=Q P_{\mu_{1}, \ldots, \mu_{n}}^{m_{1}, \ldots, m_{n}}\left(A_{1}, \ldots, A_{n}\right)
$$

откуда немедленно следует утверждение настоящего предложения.

Теперь мы рассмотрим условия, при которых выражение $f_{\mu_{1}, \ldots, \mu_{n}}\left(A_{1}, \ldots, A_{n}\right)$ обладает свойствами симметрии.

ПРЕДЛОЖЕНИЕ 2.2. Пусть $f \in \mathbb{A}\left(\left\|A_{1}\right\|, \ldots,\left\|A_{n}\right\|\right)$, әде $f\left(z_{1}, \ldots, z_{n}\right)$ задана рядом (1.1), и предположим, что существует подпоследовательность $i_{1}, \ldots, i_{\ell}$ из $1, \ldots, n$ такая, что функиия $f$ симметрична по переменным $z_{i_{1}}, \ldots, z_{i_{\ell}}$. Тогда функиия $f$ принадлежит $\mathbb{A}\left(r_{1}, \ldots, r_{n}\right)$, где $r_{i}=\left\|A_{i}\right\|$, если $i \notin\left\{i_{1}, \ldots, i_{\ell}\right\}, u$ $r_{i}=\max \left\{\left\|A_{i_{1}}\right\|, \ldots,\left\|A_{i_{\ell}}\right\|\right\}$, если $i \in\left\{i_{1}, \ldots, i_{\ell}\right\}$. Далее, любая перестановка операторов $A_{i_{1}}, \ldots, A_{i_{\ell}}$, сопровохсдемая аналогичной перестановкой мер $\mu_{i_{1}}, \ldots, \mu_{i_{\ell}}$, оставляет неизменным вырахсение $f_{\mu_{1}, \ldots, \mu_{n}}\left(A_{1}, \ldots, A_{n}\right)$. Наконеи, если $\mu_{i_{1}}=\cdots$ $=\mu_{i_{\ell}}$, то функиия $f_{\mu_{1}, \ldots, \mu_{n}}\left(A_{1}, \ldots, A_{n}\right)$ операторов $A_{1}, \ldots, A_{n}$ является симметричной функичей $A_{i_{1}}, \ldots, A_{i_{\ell}}$. 
ДокАЗАТЕЛЬСтво. Приведем доказательство для $n=2$, поскольку оно проше в смысле обозначений, и в настоящем случае это проясняет схему доказательства общего результата.

Прежде всего, без дополнительных предположений равенство

$$
P_{\mu_{1}, \mu_{2}}^{m_{1}, m_{2}}\left(A_{1}, A_{2}\right)=P_{\mu_{2}, \mu_{1}}^{m_{2}, m_{1}}\left(A_{2}, A_{1}\right)
$$

следует из определения 1.2 (как было замечено в [4, предложение 2.11]).

Далее мы заметим, что из условия симметричности $f\left(z_{2}, z_{1}\right)=f\left(z_{1}, z_{2}\right)$ следует, что $c_{m_{2}, m_{1}}=c_{m_{1}, m_{2}}$ для всех $\left(m_{1}, m_{2}\right)$. Используя также $(2.3)$, мы получаем

$$
\begin{aligned}
f_{\mu_{1}, \mu_{2}}\left(A_{1}, A_{2}\right) & =\sum_{m_{1}, m_{2}=0}^{\infty} c_{m_{1}, m_{2}} P_{\mu_{1}, \mu_{2}}^{m_{1}, m_{2}}\left(A_{1}, A_{2}\right)=\sum_{m_{1}, m_{2}=0}^{\infty} c_{m_{1}, m_{2}} P_{\mu_{2}, \mu_{1}}^{m_{2}, m_{1}}\left(A_{2}, A_{1}\right) \\
& =\sum_{m_{1}, m_{2}=0}^{\infty} c_{m_{2}, m_{1}} P_{\mu_{2}, \mu_{1}}^{m_{2}, m_{1}}\left(A_{2}, A_{1}\right)=f_{\mu_{2}, \mu_{1}}\left(A_{2}, A_{1}\right)
\end{aligned}
$$

Окончательное утверждение нашего предложения, именно, что

$$
f_{\mu_{1}, \mu_{1}}\left(A_{1}, A_{2}\right)=f_{\mu_{1}, \mu_{1}}\left(A_{2}, A_{1}\right)
$$

немедленно следует из (2.4).

Следующее предложение касается сходимости рядов в $\mathbb{D}\left(A_{1}, \ldots, A_{n}\right)$; оно имеет длинную формулировку и простое доказательство.

ПРЕДЛОЖЕНИЕ 2.3. Предположимм, что $\sum_{j=1}^{\infty} f_{j}\left(\widetilde{A}_{1}, \ldots, \widetilde{A}_{n}\right)$ является сходящимся рядом в выпутывающей алгебре $\mathbb{D}\left(A_{1}, \ldots, A_{n}\right)$, где для каждого $j$ выполнено равенство

$$
f_{j}\left(\widetilde{A}_{1}, \ldots, \widetilde{A}_{n}\right)=\sum_{m_{1}, \ldots, m_{n}=0}^{\infty} c_{m_{1}, \ldots, m_{n}}^{(j)} \widetilde{A}_{1}^{m_{1}} \ldots \widetilde{A}_{n}^{m_{n}} .
$$

Тогда, обозначая через

$$
f\left(\widetilde{A}_{1}, \ldots, \widetilde{A}_{n}\right)=\sum_{j=1}^{\infty} f_{j}\left(\widetilde{A}_{1}, \ldots, \widetilde{A}_{n}\right)
$$

сумму ряда с функиией $f$, заданной формулой (1.1), мы получим

$$
\begin{gathered}
c_{m_{1}, \ldots, m_{n}}=\sum_{j=1}^{\infty} c_{m_{1}, \ldots, m_{n}}^{(j)}, \\
\left\|f\left(\widetilde{A}_{1}, \ldots, \widetilde{A}_{n}\right)\right\|=\sum_{m_{1}, \ldots, m_{n}=0}^{\infty}\left|\sum_{j=1}^{\infty} c_{m_{1}, \ldots, m_{n}}^{(j)}\right|\left\|A_{i}\right\|^{m_{1}} \cdots\left\|A_{n}\right\|^{m_{n}} .
\end{gathered}
$$

Далее, если

$$
\sum_{m_{1}, \ldots, m_{n}=0}^{\infty}\left(\sum_{j=1}^{\infty}\left|c_{m_{1}, \ldots, m_{n}}^{(j)}\right|\right)\left\|A_{1}\right\|^{m_{1}} \cdots\left\|A_{n}\right\|^{m_{n}}<\infty
$$


то ряд в формуле (2.6) является абсолютно сходящимся в $\mathbb{D}\left(A_{1}, \ldots, A_{n}\right)$ и

$$
\begin{aligned}
\left\|f\left(\widetilde{A}_{1}, \ldots, \widetilde{A}_{n}\right)\right\| & \leqslant \sum_{j=1}^{\infty}\left\|f_{j}\left(\widetilde{A}_{1}, \ldots, \widetilde{A}_{n}\right)\right\| \\
& =\sum_{m_{1}, \ldots, m_{n}=0}^{\infty}\left(\sum_{j=1}^{\infty}\left|c_{m_{1}, \ldots, m_{n}}^{(j)}\right|\right)\left\|A_{1}\right\|^{m_{1}} \ldots\left\|A_{n}\right\|^{m_{n}} .
\end{aligned}
$$

ДоКАЗАТЕЛЬСтво. Сходящийся ряд во взвешенном $\ell_{1}$-пространстве сходится по каждой координате отдельно. Поскольку пространство $\mathbb{D}\left(A_{1}, \ldots, A_{n}\right)$ можно отождествить со взвешенным $\ell_{1}$-пространством, то соотношение $(2.8)$ справедливо. Равенство (2.9) немедленно следует из $(2.8)$ и определения нормы в $\mathbb{D}\left(A_{1}, \ldots, A_{n}\right)$. Определение нормы также обеспечивает выполнение равенства в $(2.11)$, и тогда абсолютная сходимость ряда в формуле (2.7) следует из предположения (2.10); отсюда же следует неравенство в (2.11).

Аналитические функции $n$ переменных, являющиеся тензорньми произведениями $n$ аналитических функций одной переменной, естественным образом включаются в некоторые аспекты нашей теории. Мы увидим это в этом пункте и далее в п. 4 и п. 5 . Фунцция

$$
\exp \left(z_{1}+\cdots+z_{n}\right)=\exp \left(z_{1}\right) \cdots \exp \left(z_{n}\right)
$$

будет особенно важна в связи с рядами теории возмущений (5.7) в п. 5. Ее связь с широким классом эволюционных уравнений рассматривается в [8].

ПРЕДЛОЖЕНИЕ 2.4. Пусть заданы ненулевые операторы $A_{1}, \ldots, A_{n}$ из $\mathscr{L}(X)$. Предполохим что $g_{i} \in \mathbb{A}\left(\left\|A_{i}\right\|\right)$ для $i=1, \ldots, n$, где

$$
g_{i}\left(z_{i}\right)=\sum_{m_{i}=0}^{\infty} c_{m_{i}}^{(i)} z_{i}^{m_{i}}
$$

Тогда $g_{1} \otimes \cdots \otimes g_{n} \in \mathbb{A}\left(\left\|A_{1}\right\|, \ldots,\left\|A_{n}\right\|\right) c$

$$
\left(g_{1} \otimes \cdots \otimes g_{n}\right)\left(z_{1}, \ldots, z_{n}\right):=g_{1}\left(z_{1}\right) \cdots g_{n}\left(z_{n}\right)=\sum_{m_{1}, \ldots, m_{n}=0}^{\infty} c_{m_{1}}^{(1)} \cdots c_{m_{n}}^{(n)} z_{1}^{m_{1}} \cdots z_{m}^{m_{n}}
$$

В этом случае

$$
\left(g_{1} \otimes \cdots \otimes g_{n}\right)\left(\widetilde{A}_{1}, \ldots, \widetilde{A}_{n}\right)=g_{1}\left(\widetilde{A}_{1}\right) \cdots g_{n}\left(\widetilde{A}_{n}\right)=\sum_{m_{1}, \ldots, m_{n}=0}^{\infty} c_{m_{1}}^{(1)} \cdots c_{m_{n}}^{(n)} \widetilde{A}_{1}^{m_{1}} \cdots \widetilde{A}_{n}^{m_{n}}
$$

Наконеи,

$$
\left\|\left(g_{1} \otimes \cdots \otimes g_{n}\right)\left(\widetilde{A}_{1}, \ldots, \widetilde{A}_{n}\right)\right\|=\left\|g_{1}\left(\widetilde{A}_{1}\right)\right\| \cdots\left\|g_{n}\left(\widetilde{A}_{n}\right)\right\|
$$


ДокАЗАТЕЛЬСТво. Поскольку $g_{i} \in \mathbb{A}\left(\left\|A_{i}\right\|\right)$ для $i=1, \ldots, n$, нетрудно видеть, что ряд сходится абсолютно для всех $z=\left(z_{1}, \ldots, z_{n}\right) \in \mathbb{C}^{n} \mathrm{c}\left|z_{i}\right| \leqslant\left\|A_{i}\right\|$ для $i=1, \ldots, n$, так что $g_{1} \otimes \cdots \otimes g_{n}$ принадлежит $\mathbb{A}\left(\left\|A_{1}\right\|, \ldots,\left\|A_{n}\right\|\right)$.

Второе равенство в (2.14) получается перемножением рядов для $g_{1}\left(z_{1}\right), \ldots, g_{n}\left(z_{n}\right)$. Равенства (2.15) немедленно следуют из (2.14).

Наконец, (2.16) получается, если заметить, что

$$
\begin{aligned}
\left\|\left(g_{1} \otimes \cdots \otimes g_{n}\right)\left(\widetilde{A}_{1}, \ldots, \widetilde{A}_{n}\right)\right\| & =\sum_{m_{1}, \ldots, m_{n}=0}^{\infty}\left|c_{m_{1}}^{(1)}\right| \cdots\left|c_{m_{n}}^{(n)}\right|\left\|A_{1}\right\|^{m_{1}} \cdots\left\|A_{n}\right\|^{m_{n}} \\
& =\left(\sum_{m_{1}=0}^{\infty}\left|c_{m_{1}}^{(1)}\right|\left\|A_{1}\right\|^{m_{1}}\right) \cdots\left(\sum_{m_{n}=0}^{\infty}\left|c_{m_{n}}^{(n)}\right|\left\|A_{n}\right\|^{m_{n}}\right) \\
& =\left\|g_{1}\left(\widetilde{A}_{1}\right)\right\| \cdots\left\|g_{n}\left(\widetilde{A}_{n}\right)\right\| .
\end{aligned}
$$

3. Меры, отличные от вероятностных мер. Пусть $\mu_{1}, \ldots, \mu_{n}$ - конечные, непрерьвные ненулевые меры на $\mathscr{B}[0,1]$. Как нам следует определить оператор

$$
\mathscr{T}_{\mu_{1}, \ldots, \mu_{n}} f\left(\widetilde{A}_{1}, \ldots, \widetilde{A}_{n}\right) ?
$$

Ответ: почти так же, как в определении 1.2. Это может показаться неверным, поскольку формула (1.10) предложения 1.1 вместе с эвристическими идеями Фейнмана приводит к определению 1.2, и, хотя обе части равенства (1.10) продолжают иметь смысл, равенство может быть нарушено. Простой пример показьвает это и указывает нам правильное направление. Пусть $n=1$. Тогда

$$
P^{1}\left(\widetilde{A}_{1}\right)=\widetilde{A}_{1}
$$

так же, как и ранњше, в то время как

$$
\int_{0}^{1} \widetilde{A}_{1}(s) \mu_{1}(d s)=\widetilde{A}_{1} \mu_{1}([0,1]) .
$$

Выражения в (3.1) и (3.2) совпадают, если и только если $\mu_{1}$ - вероятностная мера.

Только что приведенньй пример указьвает, что мы должны положить

$$
P_{\mu_{1}}^{1}\left(A_{1}\right)=A_{1} \mu_{1}([0,1])
$$

Но функциональное исчисление, в котором $P_{\mu_{1}}^{1}\left(A_{1}\right) \neq A_{1}$, кажется странньц. Однако можно заметить, что это естественно в случае эволюционных задач. Например, пусть $A \in \mathscr{L}(X)$. Тогда

$$
y(t)=e^{t A}=e^{\int_{0}^{t} A(s) \ell(d s)}
$$

является решением эволюционного уравнения $d y / d t=A y$ с $y(0)=I$; масса лебеговой меры $\ell$ на $[0, t]$ должна правильно учитьваться. Дальнейшие примеры рассматриваются в $[8]$. 
ОПРЕДЕЛЕНИЕ 3.1. Пусть $A_{1}, \ldots, A_{n}$ - операторы из $\mathscr{L}(X)$, и пусть $\mu_{1}, \ldots, \mu_{n}-$ конечные и непрерывные меры на $\mathscr{B}[0,1]$. Используя обозначения из определения 1.2 для любых цельх неотрицательных $m_{1}, \ldots, m_{n}$, мы определим

$$
\begin{aligned}
& \mathscr{T}_{\mu_{1}, \ldots, \mu_{n}} P^{m_{1}, \ldots, m_{n}}\left(\widetilde{A}_{1}, \ldots, \widetilde{A}_{n}\right)=P_{\mu_{1}, \ldots, \mu_{n}}^{m_{1}, \ldots, m_{n}}\left(A_{1}, \ldots, A_{n}\right) \\
& \quad:=\sum_{\pi \in S_{m}} \int_{\Delta_{m}(\pi)} C_{\pi(m)}\left(s_{\pi(m)}\right) \cdots C_{\pi(1)}\left(s_{\pi(1)}\right)\left(\mu_{1}^{m_{1}} \times \cdots \times \mu_{n}^{m_{n}}\right)\left(d s_{1}, \ldots, d s_{m}\right) .
\end{aligned}
$$

Теперь мы сделаем предположение, что все $A_{1}, \ldots, A_{n}$ и $\mu_{1}, \ldots, \mu_{n}$ ненулевые. Как мы увидим ниже, это ограничение может быть снято.

Пусть $\left\|\mu_{i}\right\|=\mu_{i}([0,1])$ для $i=1, \ldots, n$. Тогда для $f\left(\widetilde{A}_{1}, \ldots, \widetilde{A}_{n}\right) \in \mathbb{D}\left(\left\|\mu_{1}\right\| A_{1}, \ldots\right.$, $\left.\left\|\mu_{n}\right\| A_{n}\right)$, заданных формулами

$$
f\left(\widetilde{A}_{1}, \ldots, \widetilde{A}_{n}\right)=\sum_{m_{1}, \ldots, m_{n}=0}^{\infty} c_{m_{1}, \ldots, m_{n}} \widetilde{A}_{1}^{m_{1}} \ldots \widetilde{A}_{n}^{m_{n}}
$$

мы полагаем

$$
\mathscr{T}_{\mu_{1}, \ldots, \mu_{n}} f\left(\widetilde{A}_{1}, \ldots, \widetilde{A}_{n}\right):=\sum_{m_{1}, \ldots, m_{n}=0}^{\infty} c_{m_{1}, \ldots, m_{n}} \mathscr{T}_{\mu_{1}, \ldots, \mu_{n}} P^{m_{1}, \ldots, m_{n}}\left(\widetilde{A}_{1}, \ldots, \widetilde{A}_{n}\right) .
$$

ЗАМЕЧАНИЕ 3.2. Из представления (3.5) мы видим, что если существует индекс $i \in$ $\{1, \ldots, n\}$ такой, что либо $A_{i}=0$, либо $\mu_{i}=0$, то $\mathscr{T}_{\mu_{1}, \ldots, \mu_{n}} P^{m_{1}, \ldots, m_{n}}\left(\widetilde{A}_{1}, \ldots, \widetilde{A}_{n}\right)=0$ всякий раз, когда $m_{i}>0$.

Теперь рассмотрим $f$. Мынапоминаем читателю, что $f\left(\widetilde{A}_{1}, \ldots, \widetilde{A}_{n}\right) \in \mathbb{D}\left(\left\|\mu_{1}\right\| A_{1}, \ldots\right.$, $\left.\left\|\mu_{n}\right\| A_{n}\right)$ эквивалентно $f \in \mathbb{A}\left(\left\|A_{1}\right\|\left\|\mu_{1}\right\|, \ldots,\left\|A_{n}\right\|\left\|\mu_{n}\right\|\right)$. Теперь мы предположим, что либо $A_{1}=0$, либо $\mu_{1}=0$, но все $A_{2}, \ldots, A_{n}$ и $\mu_{2}, \ldots, \mu_{n}$ ненулевые. Для удобства обозначений мы полагаем $i=1$. Тогда условие на $f$ в определении 3.1 может быть сведено к предположению, что $f\left(0, z_{2}, \ldots, z_{n}\right) \in \mathbb{A}\left(\left\|A_{2}\right\|\left\|\mu_{2}\right\|, \ldots,\left\|A_{n}\right\|\left\|\mu_{n}\right\|\right)$. Заметим, что все члены ряда (3.7), для которых $m_{1}>0$, равны нулю. Аналогичное наблюдение может быть сделано в более общей ситуации.

До этого пункта мы интересовались вероятностными мерами и дополнительно предполагали, что $A_{1}, \ldots, A_{n}$ являются ненулевыми операторами. Теперь из замечания 3.2 мы видим, что последнее предположение может быть опущено.

Выше мы заметили, что условие (1.10) предложения 1.1 не выполнено, если не вьполнено требование, чтобы $\mu_{1}, \ldots, \mu_{n}$ были вероятностными мерами. Теперь мы сформулируем измененную версию предложения 1.1, которая немедленно следует из приведенного выше результата.

ПРЕДЛОЖЕНИЕ 3.3. Пусть $A_{1}, \ldots, A_{n}$ принадлежсат $\mathscr{L}(X)$. Ecли $\mu_{1}, \ldots, \mu_{n}-$ конечные непрерывные меры на $\mathscr{B}[0,1]$, то

$$
\begin{aligned}
& \left\|\mu_{1}\right\|^{m_{1}} \cdots\left\|\mu_{n}\right\|^{m_{n}} P^{m_{1}, \ldots, m_{n}}\left(\widetilde{A}_{1}, \ldots, \widetilde{A}_{n}\right) \\
& \quad=\sum_{\pi \in S_{m}} \int_{\Delta_{m}(\pi)} \widetilde{C}_{\pi(m)}\left(s_{\pi(m)}\right) \cdots \widetilde{C}_{\pi(1)}\left(s_{\pi(1)}\right)\left(\mu_{1}^{m_{1}} \times \cdots \times \mu_{n}^{m_{n}}\right)\left(d s_{1}, \ldots, d s_{m}\right) .
\end{aligned}
$$

Следующиие два предложения являются простыми, но полезными. Они описьвают способ, позволяющий распространить результаты для вероятностных мер на случай финитных мер. 
ПРЕДЛОЖЕНИЕ 3.4. Пусть $A_{1}, \ldots, A_{n}$ принадлежат $\mathscr{L}(X)$, и предположим, что $\mu_{1}, \ldots, \mu_{n}-$ финитные, непрерывные меры на $\mathscr{B}[0,1]$. Тогда

$$
\begin{aligned}
& \mathscr{T}_{\mu_{1}, \ldots, \mu_{n}} P^{m_{1}, \ldots, m_{n}}\left(\widetilde{A}_{1}, \ldots, \widetilde{A}_{n}\right) \\
& \quad=\left\|\mu_{1}\right\|^{m_{1}} \ldots\left\|\mu_{n}\right\|^{m_{n}} \mathscr{T}_{\mu_{1} /\left\|\mu_{1}\right\|, \ldots, \mu_{n} /\left\|\mu_{n}\right\|} P^{m_{1}, \ldots, m_{n}}\left(\widetilde{A}_{1}, \ldots, \widetilde{A}_{n}\right),
\end{aligned}
$$

где правая часть (3.9) интерпретируется как нулевой оператор, если одна из мер $\mu_{i}$ нулевая.

ДокАЗАТЕЛЬСтво немедленно следует из формулы (3.5) определения 3.1.

В определении 3.1 и замечании 3.2 мы ввели веса для пространств аналитических функций, отражающие полньй вес мер. Далее мы действуем в том же направлении.

ПРЕДЛОЖЕНИЕ 3.5. Пусть $A_{1}, \ldots, A_{n}$ принадлежат $\mathscr{L}(X)$, и предположим, что $\mu_{1}, \ldots, \mu_{n}$ - конечные непрерывные меры на $\mathscr{B}[0,1]$. Тогда для функиий

$$
f\left(\widetilde{A}_{1}, \ldots, \widetilde{A}_{n}\right) \in \mathbb{D}\left(\left\|\mu_{1}\right\| A_{1}, \ldots,\left\|\mu_{n}\right\| A_{n}\right),
$$

заданных формулой (3.6), мы имеем

$$
\begin{aligned}
\mathscr{T}_{\mu_{1}, \ldots, \mu_{n}} f\left(\widetilde{A}_{1}, \ldots, \widetilde{A}_{n}\right)= & f_{\mu_{1}, \ldots, \mu_{n}}\left(A_{1}, \ldots, A_{n}\right) \\
= & \sum_{m_{1}, \ldots, m_{n}=0}^{\infty} c_{m_{1}, \ldots, m_{n}}\left\|\mu_{1}\right\|^{m_{1}} \ldots\left\|\mu_{n}\right\|^{m_{n}} \\
& \quad \times \mathscr{T}_{\mu_{1} /\left\|\mu_{1}\right\|, \ldots, \mu_{n} /\left\|\mu_{n}\right\|} P^{m_{1}, \ldots, m_{n}}\left(\widetilde{A}_{1}, \ldots, \widetilde{A}_{n}\right) .
\end{aligned}
$$

ДокАЗАТЕльство. Этот результат следует из определения 3.1 и предложения 3.4 в случае, когда все $A_{1}, \ldots, A_{n}$ и $\mu_{1}, \ldots, \mu_{n}$ ненулевые; в противном случае справедливо следующее замечание.

ЗАмЕчАнИЕ 3.6. Предложение 3.4 с правильно интерпретируемой правой частью в (3.9) остается в силе, если либо $A_{i}=0$, либо $\mu_{i}=0$ для одного или более $i \in\{1, \ldots, n\}$. Действительно, согласно замечанию 3.2 ограничения на $f$ могут быть ослаблены в этом случае.

Почти все без исключения наши результаты для вероятностных мер могут быть обобщены на случай конечных мер. Когда речь идет об аналитических функциях, требуется обосновать введение веса. Доказательство формул для выпутывания является тривиальньм следствием предложения 3.4. Теперь мы рассмотрим комментарии, иллюстрирующие необходимые изменения. Подробности можно найти в [11].

(i) Веса должны быть изменены в трех частях предложения 1.4. В частности, норма правой части формулы (1.17) должна учитывать это изменение.

(ii) Формула выпутьвания (1.20) остается без изменений, как и (1.13).

(iii) В предложении 2.2 веса изменяются стандартным образом, далее мы полагаем $r_{i}=\left\|A_{i}\right\|\left\|\mu_{i}\right\|$, если $i \notin\left\{i_{1}, \ldots, i_{\ell}\right\}$, и $r_{i}=\max \left\{\left\|A_{i_{1}}\right\|\left\|\mu_{i_{1}}\right\|, \ldots,\left\|A_{i_{\ell}}\right\|\left\|\mu_{i_{\ell}}\right\|\right\}$, если $i \in\left\{i_{1}, \ldots, i_{\ell}\right\}$.

(iv) Предложения 2.3 и 2.4 не содержат выпутывания, но они используются в сочетании с конечньми мерами, не являющимися вероятностными, и поэтому требуют обоснования. Например, ряды в (2.6) должны быть сходящимися в $\mathbb{D}\left(\left\|\mu_{1}\right\| A_{1}, \ldots,\left\|\mu_{n}\right\| A_{n}\right)$. Такие изменения будут действительными и для (2.9)-(2.11). Аналогичные комментарии имеют силу для предложения 2.4 . 
ЗАмЕчАниЕ 3.7. (i) Используемые нами меры были определены на $\mathscr{B}[0,1]$, однако отрезок $[0,1]$ можно заменить на $[a, b]$.

(ii) В связи с эволюционными задачами разумным подходом является рассмотрение задач на достаточно большом фиксированном отрезке $[0, T]$ длины $T>0$. Тогда большая часть утверждений относится к отрезку $[0, t]$, где $t \in(0, T]$, а оценки часто содержат $\mu_{i}([0, t]), i=1, \ldots, n$.

(iii) Эволюционные задачи на $[0, \infty)$ рассматриваются в [8]. Основные оценки используют меры на $\mathscr{B}[0, \infty)$, которые конечны на $[0, t]$ для любого $t>0$.

Наш последний комментарий состоит в следующем. Интерес к случаю нулевых мер не является чисто алгебраическим. Существуют эволюционные задачи, в которых возмущения включаются и выключаются на некоторых отрезках времени. В этом случае полезно сузить меры на подходящие отрезки, при этом на некоторых множествах меры обращаются в нуль.

4. Влияние упорядочения носителей на формулы выпутывания. Большая часть результатов настоящего пункта легко следует из формулируемой ниже теоремы 4.1. Обозначим через $S(\mu)$ носитель меры $\mu$, т.е. дополнение множества всех точек $x$, имеюших открытую окрестность $U \in \mathscr{B}[0,1]$ такую, что $\mu(U)=0$.

Теорема 4.1. Пусть $A_{1}, \ldots, A_{n}$ принадлежст $\mathscr{L}(X)$. Предположим, что $\mu_{1}$, $\ldots, \mu_{n}$ - финитные непрерывные меры на $\mathscr{B}[0,1]$. Если существует $\xi \in(0,1)$ maкое, ито $S\left(\mu_{1}\right) \subseteq[0, \xi]$ u $S\left(\mu_{i}\right) \subseteq[\xi, 1]$ для $i=2, \ldots, n$, mо

$$
P_{\mu_{1}, \mu_{2}, \ldots, \mu_{n}}^{m_{1}, m_{2}, \ldots, m_{n}}\left(A_{1}, A_{2}, \ldots, A_{n}\right)=P_{\mu_{2}, \ldots, \mu_{n}}^{m_{2}, \ldots, m_{n}}\left(A_{2}, \ldots, A_{n}\right)\left(\mu_{1}(0,1) A_{1}\right)^{m_{1}} .
$$

ДоКАЗАТЕЛЬСТВо. В силу предположения о носителе имеем

$$
S\left[\mu_{1}^{m_{1}} \times\left(\mu_{2}^{m_{2}} \times \cdots \times \mu_{n}^{m_{n}}\right)\right] \subseteq[0, \xi]^{m_{1}} \times[\xi, 1]^{m_{2}+\cdots+m_{n}}
$$

Поэтому для любой перестановки $\pi \in S_{m}$, для которой $s_{m_{1}+1}, \ldots, s_{m}$ предшествуют $s_{1}, \ldots, s_{m_{1}}$ в списке $s_{\pi(1)}, \ldots, s_{\pi(m)}$, мы имеем

$$
\left[\mu_{1}^{m_{1}} \times\left(\mu_{2}^{m_{2}} \times \cdots \times \mu_{m}^{m_{n}}\right)\right]\left(\Delta_{m}(\pi)\right)=0 .
$$

Такие перестановки можно исключить из суммы (3.5), определяющей левую часть формулы (4.1). В интегральное выражение, отвечающее дополнению $S_{m}^{\prime}$ множества перестановок всех индексов $s_{1}, \ldots, s_{m_{1}}$, прежде всего входят $s_{m_{1}+i}, \ldots, s_{m}$ в списке $s_{\pi(1)}, \ldots$, $s_{\pi(m)}$. Поэтому во всех интегральных выражениях оператор $A_{1}^{m_{1}}$ появляется крайним справа.

Существует всего $m_{1} !\left(m_{2}+\cdots+m_{n}\right)$ ! перестановок из $S_{m}^{\prime}$, которыеможно получить, взяв любые из $m_{1}$ ! перестановок $\rho \in S_{m_{1}}$ и любые из $\left(m_{2}+\cdots+m_{n}\right)$ ! перестановок $\tau^{\prime}$ множества $m_{2}+\cdots+m_{n}$ целых чисел $m_{1}+1, \ldots, m_{1}+m_{2}+\cdots+m_{n}$. Мы обозначаем это множество перестановок через $S_{m_{1}+1, m}$. Поэтому для каждого фиксированного $\tau \in S_{m_{1}+1, m}$ каждая из $m_{1}$ ! перестановок множества $1, \ldots, m_{1}$ будет давать подьнтегральное выражение

$$
C_{\tau^{\prime}(m)}\left(S_{\tau^{\prime}(m)}\right) \cdots C_{\tau^{\prime}\left(m_{1}+1\right)}\left(s_{\tau^{\prime}\left(m_{1}+1\right)}\right) A_{1}^{m_{1}}
$$


Забегая вперед, мы также заметим, что для каждого $\rho \in S_{m_{1}}$

$$
\mu_{1}^{m_{1}}\left(\Delta_{m_{1}}(\rho)\right)=\frac{\left\|\mu_{1}\right\|^{m_{1}}}{m_{1} !} .
$$

Теперь мы можем написать

$$
\begin{aligned}
& P_{\mu_{1}, \ldots, \mu_{n}}^{m_{1}, \ldots, m_{n}}\left(A_{1}, \ldots, A_{n}\right) \\
& =\sum_{\substack{\pi \in S_{m}^{\prime} \\
\Delta_{m}(\pi)}} C_{\pi(m)}\left(s_{\pi(m)}\right) \cdots C_{\pi\left(m_{1}+1\right)}\left(s_{\pi\left(m_{1}+1\right)}\right) A_{1}^{m_{1}} \\
& \quad \times\left(\mu_{1}^{m_{1}} \times \cdots \times \mu_{n}^{m_{n}}\right)\left(d s_{1}, \ldots, d s_{m}\right) \\
& =\sum_{\substack{\tau^{\prime} \in S_{m_{1}+1, m} \\
\rho \in S_{m_{1}}}} \int_{\Delta_{m_{1}}(\rho) \times \Delta_{m_{1}+1, m}\left(\tau^{\prime}\right)} C_{\tau^{\prime}(m)}\left(s_{\tau^{\prime}(m)}\right) \cdots C_{\tau^{\prime}\left(m_{1}+1\right)}\left(s_{\tau^{\prime}\left(m_{1}+1\right)}\right) A_{1}^{m_{1}} \\
& \quad \times\left(\mu_{1}^{m_{1}} \times\left(\mu_{2}^{m_{2}} \times \cdots \times \mu_{n}^{m_{n}}\right)\right)\left(d s_{1}, \ldots, d s_{m}\right)
\end{aligned}
$$

где

$$
\Delta_{m_{1}+1, m}\left(\tau^{\prime}\right):=\left\{\left(s_{m_{1}+1}, \ldots, s_{m}\right): 0<s_{\tau^{\prime}\left(m_{1}+1\right)}<\cdots<s_{\tau^{\prime}(m)}<1\right\} .
$$

Продолжая формулу (4.6), можно получить

$$
\begin{aligned}
& P_{\mu_{1}, \ldots, \mu_{n}}^{m_{1}, \ldots, m_{n}}\left(A_{1}, \ldots, A_{n}\right) \\
& =\sum_{\tau^{\prime} \in S_{m_{1}+1, m}} \sum_{\rho \in S_{m_{1}}} \int_{\Delta_{m_{1}}(\rho) \times \Delta_{m_{1}+1, m}(\tau)} C_{\tau^{\prime}(m)}\left(s_{\tau^{\prime}(m)}\right) \cdots C_{\tau^{\prime}\left(m_{1}+1\right)}\left(s_{\tau^{\prime}\left(m_{1}+1\right)}\right) A_{1}^{m_{1}} \\
& \times\left(\mu_{1}^{m_{1}} \times\left(\mu_{2}^{m_{2}} \times \cdots \times \mu_{n}^{m_{n}}\right)\right)\left(d s_{1}, \ldots, d s_{m}\right) \\
& =\sum_{\tau^{\prime} \in S_{m_{1}+1, m}}\left\{\sum _ { \rho \in S _ { m _ { 1 } } } \left[\int_{\Delta_{m_{1}+1, m}\left(\tau^{\prime}\right)} C_{\tau^{\prime}(m)}\left(s_{\tau^{\prime}(m)}\right) \cdots C_{\tau^{\prime}\left(m_{1}+1\right)}\left(s_{\tau^{\prime}\left(m_{1}+1\right)}\right)\right.\right. \\
& \left.\left.\times\left(\mu_{2}^{m_{2}} \times \cdots \times \mu_{n}^{m_{n}}\right)\left(d s_{m_{1}+1}, \ldots, d s_{m}\right)\right] \mu_{1}^{m_{1}}\left(\Delta_{m_{1}}(\rho)\right)\right\} A_{1}^{m_{1}} \\
& =\left\{\sum_{\tau^{\prime} \in S_{m_{1}+1, m}} \int_{\Delta_{m_{1}+1, m}\left(\tau^{\prime}\right)} C_{\tau^{\prime}(m)}\left(s_{\tau^{\prime}(m)}\right) \cdots C_{\tau^{\prime}\left(m_{1}+1\right)}\left(s_{\tau^{\prime}\left(m_{1}+1\right)}\right)\right. \\
& \left.\times\left(\mu_{2}^{m_{2}} \times \cdots \times \mu_{n}^{m_{n}}\right)\left(d s_{m_{1}+1}, \ldots, d s_{m}\right)\right\}\left\{\sum_{\rho \in S_{m_{1}}} \frac{\left\|\mu_{1}\right\|^{m_{1}}}{m_{1} !}\right\} A_{1}^{m_{1}} \\
& =\left\{\sum_{\tau \in S_{m_{2}+\cdots+m_{n}}} \int_{\Delta_{m_{2}+\cdots+m_{n}}} C_{\tau\left(m_{2}+\cdots+m_{n}\right)}\left(s_{\tau\left(m_{2}+\cdots+m_{n}\right)}\right) \cdots C_{\tau(1)}\left(s_{\tau(1)}\right)\right. \\
& \left.\times\left(\mu_{2}^{m_{2}} \times \cdots \times \mu_{n}^{m_{n}}\right)\left(d s_{1}, \ldots, d s_{m_{2}}+\cdots+m_{n}\right)\right\}\left(\left\|\mu_{1}\right\| A_{1}\right)^{m_{1}},
\end{aligned}
$$

где $\tau(j)=\tau^{\prime}\left(m_{1}+j\right)$ для $j=1, \ldots, m_{2}+\cdots+m_{n}$. Теперь равенство (4.1) следует из (4.8). 
ЗАмечаниЕ 4.2. (i) Тот факт, что носитель меры $\mu_{1}$ в теореме 4.1 был слева от носителей всех остальных мер, исключительно удобен, но не принципиален для доказательства. Если носитель $\mu_{j}$ находится слева от носителей остальных мер, то

$$
\begin{aligned}
& P_{\mu_{1}, \ldots, \mu_{n}}^{m_{1}, \ldots, m_{n}}\left(A_{1}, \ldots, A_{n}\right) \\
& \quad=P_{\mu_{1}, \ldots, \mu_{j-1}, \mu_{j+1}, \ldots, \mu_{n}}^{m_{1}, \ldots, m_{j-1}, m_{j+1}, \ldots, m_{n}}\left(A_{1}, \ldots, A_{j-1}, A_{j+1}, \ldots, A_{n}\right)\left(\left\|\mu_{j}\right\| A_{j}\right)^{m_{j}}
\end{aligned}
$$

(ii) Если мера $\mu_{j}$ в части (i) этого замечания имеет носитель справа от носителей остальных мер, то вместо (4.9) имеем

$$
\begin{aligned}
& P_{\mu_{1}, \ldots, \mu_{n}}^{m_{1}, \ldots, m_{n}}\left(A_{1}, \ldots, A_{n}\right) \\
& \quad=\left(\left\|\mu_{j}\right\| A_{j}\right)^{m_{j}} P_{\mu_{1}, \ldots, \mu_{j-1}, \mu_{j+1}, \ldots, \mu_{n}}^{m_{1}, \ldots, m_{j-1}, m_{j+1}, \ldots, m_{n}}\left(A_{1}, \ldots, A_{j-1}, A_{j+1}, \ldots, A_{n}\right)
\end{aligned}
$$

Используя теорему 4.1, замечание 4.2 и метод индукции, мы легко получаем следующее утверждение.

СлЕДСТВИЕ 4.3. Пусть $A_{1}, \ldots, A_{n}$ принадлежат алгебре $\mathscr{L}(X)$, и предположим, что $\mu_{1}, \ldots, \mu_{n}$ - конечные непрерывные меры на $\mathscr{B}[0,1]$. Пусть $\left\{i_{1}, \ldots, i_{k}\right\}$ является подмножеством множества $\{1, \ldots, n\}$, и предположим, что носители соответствуюших мер упорядочены правильно:

$$
S\left(\mu_{i_{1}}\right) \leqslant S\left(\mu_{i_{2}}\right) \leqslant \cdots \leqslant S\left(\mu_{i_{k}}\right)
$$

В обозначениях

$$
\{1, \ldots, n\} \backslash\left\{i_{1}, \ldots, i_{k}\right\}=\left\{j_{1}, \ldots, j_{n-k}\right\}, \quad j_{1}<\cdots<j_{n-k}
$$

мы предполагаем, что

$$
S\left(\mu_{i_{k}}\right) \leqslant S\left(\mu_{j_{\ell}}\right), \quad \ell=1, \ldots, n-k .
$$

Тогда

$$
\begin{aligned}
& P_{\mu_{1}, \ldots, \mu_{n}}^{m_{1}, \ldots, m_{n}}\left(A_{1}, \ldots, A_{n}\right) \\
& \quad=P_{\mu_{j_{1}}, \ldots, \mu_{j_{n-k}}}^{m_{j_{1}}, \ldots, m_{j_{n-k}}}\left(A_{j_{1}}, \ldots, A_{j_{n-k}}\right)\left(\left\|\mu_{i_{k}}\right\| A_{i_{k}}\right)^{m_{i_{k}}} \ldots\left(\left\|\mu_{i_{1}}\right\| A_{i_{1}}\right)^{m_{i_{1}}} .
\end{aligned}
$$

В частности, если $k=n$, то

$$
P_{\mu_{1}, \ldots, \mu_{n}}^{m_{1}, \ldots, m_{n}}\left(A_{1}, \ldots, A_{n}\right)=\left(\left\|\mu_{i_{n}}\right\| A_{i_{n}}\right)^{m_{i_{n}}} \ldots\left(\left\|\mu_{i_{1}}\right\| A_{i_{1}}\right)^{m_{i}}
$$

Если неравенства, используемые для упорядочения носителей в формулах (4.11) $u$ (4.13), изменены на противоположсные, то порядок операторов в правой части (4.14) также меняется на противополохний.

Такое же утверждение верно для правой части (4.15), но “новая" версия (4.15) является лишь старым результатом в новых обозначениях. 
СлЕДСТВИЕ 4.4. Пусть предположсения первой части следствия 4.3 выполнень, включая предположения (4.11) и (4.13), а также включая упорядочение носителей. Тогда для любых $f\left(\widetilde{A}_{1}, \ldots, \widetilde{A}_{n}\right) \in \mathbb{D}\left(\left\|\mu_{1}\right\| A_{1}, \ldots,\left\|\mu_{n}\right\| A_{n}\right)$ таких, ито $f$ является әлементом банаховой алгебры $\mathbb{A}\left(\left\|A_{1}\right\|\left\|\mu_{1}\right\|, \ldots,\left\|A_{n}\right\|\left\|\mu_{n}\right\|\right)$ функичй (1.1), имеем

$$
\begin{aligned}
\mathscr{T}_{\mu_{1}, \ldots, \mu_{n}} f\left(\widetilde{A}_{1}, \ldots, \widetilde{A}_{n}\right)= & f_{\mu_{1}, \ldots, \mu_{n}}\left(A_{1}, \ldots, A_{n}\right) \\
= & \sum_{\substack{m_{1}, \ldots, m_{n}=0\\
}}^{\infty} c_{m_{1}, \ldots, m_{n}} P_{\mu_{j_{1}}, \ldots, \mu_{j_{n-k}}}^{m_{j_{1}}, \ldots, m_{j_{n-k}}}\left(A_{j_{1}}, \ldots, A_{j_{n-k}}\right) \\
& \times\left(\left\|\mu_{i_{k}}\right\| A_{i_{k}}\right)^{m_{i_{k}}} \ldots\left(\left\|\mu_{i_{1}}\right\| A_{i_{1}}\right)^{m_{i_{1}}} .
\end{aligned}
$$

В частности, если $k=n$ такое, что носители мер полностью упорядочены $u$ условие (4.15) выполнено, то

$$
\begin{aligned}
\mathscr{T}_{\mu_{1}, \ldots, \mu_{n}} f\left(\widetilde{A}_{1}, \ldots, \widetilde{A}_{n}\right) & =f_{\mu_{1}, \ldots, \mu_{n}}\left(A_{1}, \ldots, A_{n}\right) \\
& =\sum_{m_{1}, \ldots, m_{n}=0}^{\infty} c_{m_{1}, \ldots, m_{n}}\left(\left\|\mu_{i_{n}}\right\| A_{i_{n}}\right)^{m_{i_{n}}} \ldots\left(\left\|\mu_{i_{1}}\right\| A_{i_{1}}\right)^{m_{i_{1}}}
\end{aligned}
$$

Наконеи, если неравенства в (4.11) и (4.13), используемые для упорядочения, изменить на обратные, то порядок операторов в каждом члене правой части (4.16) также изменится на обратный.

Если функция $f$ является тензорным произведением $n$ функций одной комплексной переменной, то результаты следствия 4.4 имеют особенно красивую форму. Эта ситуация рассматривается ниже, именно в случае $n=k$ из предыдущего следствия.

ПРЕДЛОЖЕНИЕ 4.5. Пусть $A_{1}, \ldots, A_{n}$ принадлежат $\mathscr{L}(X)$, и предположим, что $\mu_{1}, \ldots, \mu_{n}-ф и н и т н ы е ~ н е п р е р ы в н ы е ~ м е р ы ~ н а ~ \mathscr{B}[0,1]$ такие, что

$$
S\left(\mu_{i_{1}}\right) \leqslant S\left(\mu_{i_{2}}\right) \leqslant \cdots \leqslant S\left(\mu_{i_{n}}\right)
$$

әде $i_{1}, \ldots, i_{n}$ - некоторая перестановка индексов $1, \ldots, n$. Далее предположим, что кажсдая функиия $g_{i}$ принадлежит $\mathbb{A}\left(\left\|A_{i}\right\|\left\|\mu_{i}\right\|\right)$, где $g_{i}$ заданы формулами (2.13) для $i=1, \ldots, n$. Тогда

$$
\begin{aligned}
\mathscr{T}_{\mu_{1}, \ldots, \mu_{n}}\left(g_{1} \otimes \cdots \otimes g_{n}\right)\left(\widetilde{A}_{1}, \ldots, \widetilde{A}_{n}\right) & =\left(g_{1} \otimes \cdots \otimes g_{n}\right)_{\mu_{1}, \ldots, \mu_{n}}\left(A_{1}, \ldots, A_{n}\right) \\
& =g_{i_{n}}\left(\left\|\mu_{i_{n}}\right\| A_{i_{n}}\right) \cdots g_{i_{1}}\left(\left\|\mu_{i_{1}}\right\| A_{i_{1}}\right) .
\end{aligned}
$$

В частности,

$$
\exp _{\mu_{1}, \ldots, \mu_{n}}\left(A_{1}, \ldots, A_{n}\right)=\exp \left(\left\|\mu_{i_{n}}\right\| A_{i_{n}}\right) \cdots \exp \left(\left\|\mu_{i_{1}}\right\| A_{i_{1}}\right)
$$


ДоКАЗАТЕльство. Этот результат легко следует из следствия 4.4 и предложения 2.4 применительно к случаю финитных мер. Именно, применяя (4.17) и (2.14), мы имеем

$$
\begin{aligned}
\left(g_{1}\right. & \left.\otimes \cdots \otimes g_{n}\right)_{\mu_{1}, \ldots, \mu_{n}}\left(A_{1}, \ldots, A_{n}\right) \\
& =\sum_{m_{1}, \ldots, m_{n}=0}^{\infty} c_{m_{1}}^{(1)} \cdots c_{m_{n}}^{(n)}\left(\left\|\mu_{i_{n}}\right\| A_{i_{n}}\right)^{m_{i_{n}}} \cdots\left(\left\|\mu_{i_{1}}\right\| A_{i_{1}}\right)^{m_{1}} \\
& =\left[\sum_{m_{i_{n}}=0}^{\infty} c_{m_{i_{m}}}^{\left(i_{n}\right)}\left(\left\|\mu_{i_{n}}\right\| A_{i_{n}}\right)^{m_{i_{n}}}\right] \cdots\left[\sum_{m_{i_{1}}=0}^{\infty} c_{m_{i_{1}}}^{\left(i_{1}\right)}\left(\left\|\mu_{i_{1}}\right\| A_{i_{1}}\right)^{m_{i_{1}}}\right] \\
& =g_{i_{n}}\left(\left\|\mu_{i_{n}}\right\| A_{i_{n}}\right) \cdots g_{i_{1}}\left(\left\|\mu_{i_{1}}\right\| A_{i_{1}}\right),
\end{aligned}
$$

что совпадает с (4.19). Формула (4.20) является специальным частным случаем (4.19).

Уравнение (4.19) есть простая формула выпутывания, которая имеет место для функций, являющихся тензорами при условии (4.18) на носители. Предложение 4.5 распространяется без значительных усложнений на случай функций, являющихся конечными или бесконечными суммами тензорных произведений.

ПРЕДЛОЖЕНИЕ 4.6. Пусть $A_{1}, \ldots, A_{n} u \mu_{1}, \ldots, \mu_{n}$ удовлетворяют предположениям предьдущего предложения, и пусть для каждого $j=1,2, \ldots$ и $i=1, \ldots, n$ выполнено $g_{i, j} \in \mathbb{A}\left(\left\|A_{i}\right\|\left\|\mu_{i}\right\|\right)$, так ито $g_{1, j} \otimes \cdots \otimes g_{n, j} \in \mathbb{A}\left(\left\|A_{1}\right\|\left\|\mu_{1}\right\|, \ldots,\left\|A_{n}\right\|\left\|\mu_{n}\right\|\right)$. Наконеи, предположим, что ряд

$$
\sum_{j=1}^{\infty} g_{1, j} \otimes \cdots \otimes g_{n, j}
$$

сходится в $\mathbb{A}\left(\left\|A_{1}\right\|\left\|\mu_{1}\right\|, \ldots,\left\|A_{n}\right\|\left\|\mu_{n}\right\|\right)$. Тогда

$$
\begin{aligned}
\mathscr{T}_{\mu_{1}, \ldots, \mu_{n}}\left[\sum_{j=1}^{\infty}\left(g_{1, j} \otimes \cdots \otimes g_{n, j}\right)\left(\widetilde{A}_{1}, \ldots, \widetilde{A}_{n}\right)\right] \\
=\left(\sum_{j=1}^{\infty} g_{1, j} \otimes \cdots \otimes g_{n, j}\right)_{\mu_{1}, \ldots, \mu_{n}}\left(A_{1}, \ldots, A_{n}\right) \\
=\sum_{j=1}^{\infty} g_{i_{n}, j}\left(\left\|\mu_{i_{n}}\right\| A_{i_{n}}\right) \cdots g_{i_{1}, j}\left(\left\|\mu_{i_{1}}\right\| A_{i_{1}}\right) .
\end{aligned}
$$

ДокАЗАТЕЛЬСТВо. Достаточно использовать предложение 4.5 и непрерывность отображения $\mathscr{T}_{\mu_{1}, \ldots, \mu_{n}}$.

Ряд (4.22) абсолютно сходится в пространстве $\mathbb{A}\left(\left\|A_{1}\right\|\left\|\mu_{1}\right\|, \ldots,\left\|A_{n}\right\|\left\|\mu_{n}\right\|\right)$ при условии, что $\sum_{j=1}^{\infty}\left\|g_{1, j}\right\| \cdots\left\|g_{n, j}\right\|<\infty$.

Далеемы покажем, что произведение Троттера (формулу произведения Троттера см. в [12, теорема VIII.30]) может быть получено, используя предложение 4.5.

Пусть $\mu_{i}, i=1, \ldots, 2 n$, является мерой Лебега на интервале $[(i-1) /(2 n), i /(2 n)]$ и нулем на дополнении этого множества в $[0,1]$. Пусть также заданы $B_{1}, B_{2}$, принадлежашие $\mathscr{L}(X)$, и пусть

$$
A_{i}= \begin{cases}B_{1}, & \text { если } i \text { нечетно, } \\ B_{2}, & \text { если } i \text { четно. }\end{cases}
$$


Тогда из (4.20) предложения 4.5 имеем

$$
\begin{aligned}
\exp _{\mu_{1}, \ldots, \mu_{2 n}}\left(A_{1}, \ldots, A_{2 n}\right) & =\exp \left(\frac{1}{2 n} A_{2 n}\right) \exp \left(\frac{1}{2 n} A_{2 n-1}\right) \cdots \exp \left(\frac{1}{2 n} A_{2}\right) \exp \left(\frac{1}{2 n} A_{1}\right) \\
& =\left[\exp \left(\frac{1}{2} \frac{B_{2}}{n}\right) \exp \left(\frac{1}{2} \frac{B_{1}}{n}\right)\right]^{n} .
\end{aligned}
$$

Но последнее выражение в (4.24) является в точности аппроксимацией $n$-го порядка формулы произведения Троттера для $\exp \left(\left(B_{1}+B_{2}\right) / 2\right)$. Заметим также, что произведения Троттера существенно используются в [10].

Основной результат настоящего пункта предполагает, что существует по крайней мере одна мера, носитель которой лежит слева (или справа) от носителей других мер. Мы приводим здесь краткое описание примера, в котором формула вьпутывания является более сложной.

ПримеР 4.7. Пусть $A_{1}, A_{2}, A_{3}$ принадлежат $\mathscr{L}(X)$, и пусть $\mu_{1}, \mu_{2}, \mu_{3}$ определены на $\mathscr{B}[0,1]$. Предположим, что $S\left(\mu_{1}\right) \subseteq[1 / 3,2 / 3]$ и $S\left(\mu_{i}\right) \subseteq[0,1 / 3] \cup[2 / 3,1]$ для $i=2,3$. Тогда $S\left(\mu_{1} \times \mu_{2} \times \mu_{3}\right)$ является подмножеством объединения следующих непересекающихся множеств:

$$
\left[\frac{1}{3}, \frac{2}{3}\right] \times\left[0, \frac{1}{3}\right]^{2}, \quad\left[\frac{1}{3}, \frac{2}{3}\right] \times\left[0, \frac{1}{3}\right] \times\left[\frac{2}{3}\right], \quad\left[\frac{1}{3}, \frac{2}{3}\right] \times\left[\frac{2}{3}, 1\right] \times\left[0, \frac{1}{3}\right],\left[\frac{1}{3}\right] \times\left[\frac{2}{3}, 1\right]^{2} .
$$

Рассматривая, что проиходит на каждом из этих множеств, можно видеть, что

$$
\begin{aligned}
& P_{\mu_{1}, \mu_{2}, \mu_{3}}^{1,1,1}\left(A_{1}, A_{2}, A_{3}\right)=\left(\left\|\mu_{1}\right\| A_{1}\right)\left\{A_{3} A_{2}\left(\mu_{2} \times \mu_{3}\right)\left(\left[0<s_{2}<s_{3}<\frac{1}{3}\right]\right)\right. \\
& \left.+A_{2} A_{3}\left(\mu_{2} \times \mu_{3}\right)\left(\left[0<s_{3}<s_{2}<\frac{1}{3}\right]\right)\right\}+\left(\mu_{3}\left[\frac{2}{3}, 1\right] A_{3}\right)\left(\left\|\mu_{1}\right\| A_{1}\right)\left(\mu_{2}\left[0, \frac{1}{3}\right] A_{2}\right) \\
& \quad+\left(\mu_{2}\left[\frac{2}{3}, 1\right] A_{2}\right)\left(\left\|\mu_{1}\right\| A_{1}\right)\left(\mu_{3}\left[0, \frac{1}{3}\right] A_{3}\right)+\left\{A_{2} A_{3}\left(\mu_{2} \times \mu_{3}\right)\left(\left[\frac{2}{3}<s_{3}<s_{2}<1\right]\right)\right. \\
& \left.\quad+A_{3} A_{2}\left(\mu_{2} \times \mu_{3}\right)\left(\left[\frac{2}{3}<s_{2}<s_{3}<1\right]\right)\right\}\left(\left\|\mu_{1}\right\| A_{1}\right) .
\end{aligned}
$$

Как было замечено во введении, результаты настоящего пункта связьвают теорию, развиваемую авторами в настоящей работе и в [4], [5], [10], с элементарными аспектами работ [6] и [7].

5. Выпутывание экспоненциального множителя. Фейнман рассматривал свою формулу вьпутывания "экспериментального", точнее, экспоненциального множителя как основной результат работы [1]. В этом пункте мы приводим математически строгий вывод этой формулы. На самом деле, основной результат - приводимая ниже теорема 5.1 - допускает разнообразные меры и не ограничивается случаем экспоненциальных функций. Варьирование множества мер означает, что мы можем рассматривать одновременно различные операторные исчисления.

Пусть $A, B_{1}, \ldots, B_{k}$ - элементы алгебры $\mathscr{L}(X)$ и $m, n_{1}, \ldots, n_{k}$ - неотрицательные целые числа. Сначала мы “выпутаем" $\widetilde{A}^{m} \widetilde{B}_{1}^{n_{1}} \cdots \widetilde{B}_{k}^{n_{k}}$. Идея состоит в том, чтобы выделить члены, соответствуюшие $\widetilde{A}$, из других членов. 
Пусть $n=n_{1}+\cdots+n_{k}$. Предположим, что $\mu$ и $\nu_{1}, \ldots, \nu_{k}-$ непрерьвные вероятностные меры на борелевской $\sigma$-алгебре $\mathscr{B}[0,1]$ на отрезке $[0,1]$. Согласно определению 1.2 и (1.13) вьпутьвание множителя $P_{\mu, \nu_{1}, \ldots, \nu_{k}}^{m, n_{1}, \ldots, n_{k}}\left(A, B_{1}, \ldots, B_{k}\right)$ из $\widetilde{A}^{m} \widetilde{B}_{1}^{n_{1}} \cdots \widetilde{B}_{k}^{n_{k}}$ дается выражением

$$
\begin{gathered}
\sum_{\pi \in S_{m+n}} \int_{\Delta_{m+n}(\pi)} C_{\pi(m+n)}\left(s_{\pi(m+n)}\right) \cdots C_{\pi(1)}\left(s_{\pi(1)}\right) \\
\times\left(\mu^{m} \times \nu_{1}^{n_{1}} \times \cdots \times \nu_{k}^{n_{k}}\right)\left(d s_{1}, \ldots, d s_{m+n}\right)
\end{gathered}
$$

где

$$
C_{i}(s)=\left\{\begin{array}{l}
A, \quad \text { если } i \in\{1, \ldots, m\}, \\
B_{1}, \text { если } i \in\left\{m+1, \ldots, m+n_{1}\right\}, \\
B_{2}, \text { если } i \in\left\{m+n_{1}+1, \ldots, m+n_{1}+n_{2}\right\}, \\
\ldots \ldots \ldots \ldots \ldots \ldots \ldots \ldots \ldots \ldots \\
B_{k}, \text { если } i \in\left\{m+n_{1}+\cdots \ldots+n_{k-1}+1, \ldots, m+n\right\},
\end{array}\right.
$$

для всех $0 \leqslant s \leqslant 1$. Как обычно, в наших обозначениях мы опускаем зависимость от $m, n_{1}, \ldots, n_{k}$.

Выражение (5.1) может быть записано явно в терминах $A$ и $B_{1}, \ldots, B_{k}$. Для $\pi \in S_{m+n}$ рассмотрим множество $J_{\pi}=\pi^{-1}(\{m+1, \ldots, m+n\})$. Тогда $C_{\pi(j)}(s) \in\left\{B_{1}, \ldots, B_{k}\right\}$ для всех $0 \leqslant s \leqslant 1$ и всех $j \in J_{\pi}$ и $C_{\pi(j)}(s)=A$ для всех $0 \leqslant s \leqslant 1$ и всех $j \notin J_{\pi}$, $j=1, \ldots, m+n$.

Предположим, что $J_{\pi}=\left\{u_{1}, \ldots, u_{n}\right\}$ для целых $n$ чисел $u_{1}<\cdots<u_{n}$ от 1 до $m+n$ и для каждого $k=0, \ldots, n$ сушествует целое $j_{k}$ большее, чем $u_{k}$, и меньшее, чем $u_{k+1}$. Здесь мы полагаем $u_{0}=0$ и $u_{n+1}=m+n+1$, т.е.

$$
j_{0}=u_{1}-1, \quad j_{1}=u_{2}-u_{1}-1, \quad \ldots, \quad j_{n-1}=u_{n}-u_{n-1}-1, \quad j_{n}=m+n-u_{n} .
$$

Для каждого $\left(s_{1}, \ldots, s_{m+n}\right) \in \Delta_{m+n}(\pi)$ мы имеем

$$
C_{\pi(m+n)}\left(s_{\pi(m+n)}\right) \cdots C_{\pi(1)}\left(s_{\pi(1)}\right)=A^{j_{n}} B_{\pi\left(u_{n}\right)} A^{j_{n-1}} \cdots A^{j_{1}} B_{\pi\left(u_{1}\right)} A^{j_{0}} .
$$

Тогда интеграл

$$
\int_{\Delta_{m+n}(\pi)} C_{\pi(m+n)}\left(s_{\pi(m+n)}\right) \cdots C_{\pi(1)}\left(s_{\pi(1)}\right)\left(\mu^{m} \times \nu_{1}^{n_{1}} \times \cdots \times \nu_{k}^{n_{k}}\right)\left(d s_{1}, \ldots, d s_{m+n}\right)
$$

преобразуется к виду

$$
\left(\mu^{m} \times \nu_{1}^{n_{1}} \times \cdots \times \nu_{k}^{n_{k}}\right)\left(\Delta_{m+n}(\pi)\right) A^{j_{n}} B_{\pi\left(u_{n}\right)} A^{j_{n-1}} \cdots A^{j_{1}} B_{\pi\left(u_{1}\right)} A^{j_{0}} .
$$

В силу приведенной выше конструкции каждой перестановке $\pi \in S_{m+n}$ соответствуют неотрицательные целые $\left(j_{0}, \ldots, j_{n}\right)$ такие, что $j_{0}+\cdots+j_{n}=m$. Обратно, целые числа $\left(j_{0}, \ldots, j_{n}\right)$ однозначно определяют подмножества с $n$ элементами $J \subset\{1, \ldots, m+n\}$. Существует $m ! n !$ перестановок $\pi \in S_{m+n}$ таких, что $\pi^{-1}(\{m+1, \ldots, m+n\})=J$, соответствующих множеству $\mathscr{B}_{j_{0}, \ldots, j_{n}}$ из $n$ ! биекций из $J$ на $\{m+1, \ldots, m+n\}$ и множества $\mathscr{A}_{j_{0}, \ldots, j_{n}}$ из $m$ ! биекций из $\{1, \ldots, m+n\} \backslash J$ на $\{1, \ldots, m\}$. 
Пусть $j_{0}, \ldots, j_{n}$ - неотрицательные целые числа такие, что $j_{0}+\cdots+j_{n}=m$, и пусть $J=\left\{u_{1}, \ldots, u_{n}\right\}$ - набор соответствующих $n$ целых чисел $u_{1}<\cdots<u_{n}$ от 1 до $m+n$. Пусть $\beta \in \mathscr{B}_{j_{0}, \ldots, j_{n}}$ и $\alpha \in \mathscr{A}_{j_{0}, \ldots, j_{n}}$.

Для каждого $0 \leqslant u<v \leqslant 1$ и каждого целого $k=0,1, \ldots, n$ положим

$$
\begin{aligned}
\Delta_{j_{0}, \ldots, j_{n}}^{\alpha}[u, v ; k] & =\left\{\left(s_{1}, \ldots, s_{m}\right) \in \mathbb{R}^{m}: u \leqslant s_{\alpha\left(u_{k}+1\right)}<\cdots<s_{\alpha\left(u_{k+1}-1\right)} \leqslant v\right\}, \\
\Gamma_{j_{0}, \ldots, j_{n}}^{\beta} & =\left\{\left(s_{m+1}, \ldots, s_{m+n}\right) \in \mathbb{R}^{n}: 0 \leqslant s_{\beta\left(u_{1}\right)}<\cdots<s_{\beta\left(u_{n}\right)} \leqslant 1\right\} .
\end{aligned}
$$

Целые числа $j_{0}, \ldots, j_{n}$ и отображения $\beta \in \mathscr{B}_{j_{0}, \ldots, j_{n}}$ и $\alpha \in \mathscr{A}_{j_{0}, \ldots, j_{n}}$ однозначно определяют перестановку $\pi \in S_{m+n}$, для которой

$$
\Delta_{m+n}(\pi) \equiv \bigcup_{s \in \Gamma_{j_{0}, \ldots, j_{n}}^{\beta}}\left(\Delta_{j_{0}, \ldots, j_{n}}^{\alpha}\left[0, s_{\beta\left(u_{1}\right)} ; 0\right] \cap \cdots \cap \Delta_{j_{0}, \ldots, j_{n}}^{\alpha}\left[s_{\beta\left(u_{n}\right)}, 1 ; n\right]\right) \times\{s\} .
$$

Использование теоремы Фубини показьвает, что мера

$$
\left(\mu^{m} \times \nu_{1}^{n_{1}} \times \cdots \times \nu_{k}^{n_{k}}\right)\left(\Delta_{m+n}(\pi)\right)
$$

множества $\Delta_{m+n}(\pi)$, т.е. числовой множитель в $(5.2)$, равна

$$
\begin{aligned}
& \int_{\Gamma_{j_{0}, \ldots, j_{n}}^{\beta}} \mu^{m}\left(\Delta_{j_{0}, \ldots, j_{n}}^{\alpha}\left[0, s_{\beta\left(u_{1}\right)} ; 0\right] \cap \cdots \cap \Delta_{j_{0}, \ldots, j_{n}}^{\alpha}\left[s_{\beta\left(u_{n}\right)}, 1 ; n\right]\right) \\
& \quad \times\left(\nu_{1}^{n_{1}} \times \cdots \times \nu_{k}^{n_{k}}\right)\left(d s_{m+1}, \ldots, d s_{m+n}\right),
\end{aligned}
$$

где $\mu^{0}(\varnothing)=1$. Продакт-мера $\mu^{m}$ инвариантна относительно действия перестановок индексов, поэтому для фиксированного $\beta \in \mathscr{B}_{j_{0}}, \ldots, j_{n}$ это число одинаково для всех $\alpha \in \mathscr{A}_{j_{0}, \ldots, j_{n}}$.

Выражение (5.3) может быть переписано следующим образом:

$$
\begin{aligned}
& \int_{\Gamma_{j_{0}, \ldots, j_{n}}^{\beta}} \mu^{j_{0}}\left(\Delta_{j_{0}}\left[0, s_{\beta\left(u_{1}\right)}\right]\right) \mu^{j_{1}}\left(\Delta_{j_{1}}\left[s_{\beta\left(u_{1}\right)}, s_{\beta\left(u_{2}\right)}\right]\right) \cdots \mu^{j_{n}}\left(\Delta_{j_{n}}\left[s_{\beta\left(u_{n}\right)}, 1\right]\right) \\
& \quad \times\left(\nu_{1}^{n_{1}} \times \cdots \times \nu_{k}^{n_{k}}\right)\left(d s_{m+1}, \ldots, d s_{m+n}\right) \\
& =\frac{1}{j_{0} ! \cdots j_{n} !} \int_{\Gamma_{j_{0}, \ldots, j_{n}}^{\beta}} \mu\left(\left[0, s_{\beta\left(u_{1}\right)}\right]\right)^{j_{0}} \mu\left(\left[s_{\beta\left(u_{1}\right)}, s_{\beta\left(u_{2}\right)}\right]\right)^{j_{1}} \cdots \mu\left(\left[s_{\beta\left(u_{n}\right)}, 1\right]\right)^{j_{n}} \\
& \quad \times\left(\nu_{1}^{n_{1}} \times \cdots \times \nu_{k}^{n_{k}}\right)\left(d s_{m+1}, \ldots, d s_{m+n}\right) .
\end{aligned}
$$

Множество $\mathscr{B}_{j_{0}}, \ldots, j_{n}$ находится во взаимно однозначном соответствии с $S_{n}$. Обозначим

$$
(\overbrace{B_{1}, \ldots, B_{1}}^{n_{1}}, \ldots, \overbrace{B_{j}, \ldots, B_{j}}^{n_{j}}, \ldots, \overbrace{B_{k}, \ldots, B_{k}}^{n_{k}})
$$

через $\left(B_{1}^{\prime}, \ldots, B_{n}^{\prime}\right)$. Сумма выражений (5.2) по всем $\pi \in S_{m+n}$ равна

$$
\sum_{j_{0}+\cdots+j_{n}=m} \sum_{\pi \in S_{n}} \frac{m !}{j_{0} ! \ldots j_{n} !} \gamma_{j_{0}, \ldots, j_{n}}(\pi) A^{j_{n}} B_{\pi(n)}^{\prime} A^{j_{n-1}} \cdots A^{j_{1}} B_{\pi(1)}^{\prime} A^{j_{0}},
$$

где для всех неотрицательных целых $j_{0}, \ldots, j_{n}$ и $\pi \in S_{n}$ число $\gamma_{j_{0}}, \ldots, j_{n}(\pi)$ равно интегралу

$$
\int_{\Delta_{n}(\pi)} \mu\left(\left[0, s_{\pi(1)}\right]\right)^{j_{0}} \mu\left(\left[s_{\pi(1)}, s_{\pi(2)}\right]\right)^{j_{1}} \cdots \mu\left(\left[s_{\pi(n)}, 1\right]\right)^{j_{n}}\left(\nu_{1}^{n_{1}} \times \cdots \times \nu_{k}^{n_{k}}\right)\left(d s_{1}, \ldots, d s_{n}\right) .
$$

Таким образом, мы доказали следующее утверждение. 
Теорема 5.1. Пусть $k$ - натуральное, и пусть $\mu$ и $\nu_{1} \ldots, \nu_{k}$ - непрерывные вероятностные меры на борелевской $\sigma$-алгебре $\mathscr{B}([0,1])$. Пусть $A, B_{1}, \ldots, B_{k}-$ әлементы из $\mathscr{L}(X)$. Предположим, что $f \in \mathbb{A}\left(\|A\|,\left\|B_{1}\right\|, \ldots,\left\|B_{k}\right\|\right)$ заданы следующим образом:

$$
f\left(z_{0}, \ldots, z_{k}\right)=\sum_{m, n_{1}, \ldots, n_{k}=0}^{\infty} c_{m, n_{1}, \ldots, n_{k}} z_{0}^{m} z_{1}^{n_{1}} \cdots z_{k}^{n_{k}}
$$

Tогда

$$
\begin{aligned}
& f_{\mu, \nu_{1}, \ldots, \nu_{k}}\left(A, B_{1}, \ldots, B_{k}\right) \\
& =\sum_{m, n_{1}, \ldots, n_{k}=0}^{\infty} c_{m, n_{1}, \ldots, n_{k}} m !\left(\sum_{j_{0}+\cdots+j_{n}=m} \sum_{\pi \in \mathscr{S}_{n}} \frac{1}{j_{0} ! \cdots j_{n} !} \gamma_{j_{0}, \ldots, j_{n}}(\pi)\right. \\
& \left.\quad \times A^{j_{n}} B_{\pi(n)}^{\prime} A^{j_{n-1}} \cdots A^{j_{1}} B_{\pi(1)}^{\prime} A^{j_{0}}\right)
\end{aligned}
$$

где для кажсдого $j_{0}, \ldots, j_{n} u \pi \in S_{n}$ число $\gamma_{j_{0}, \ldots, j_{n}}(\pi)$ равно интегралу

$$
\int_{\Delta_{n}(\pi)} \mu\left(\left[0, s_{\pi(1)}\right]\right)^{j_{0}} \mu\left(\left[s_{\pi(1)}, s_{\pi(2)}\right]\right)^{j_{1}} \cdots \mu\left(\left[s_{\pi(n)}, 1\right]\right)^{j_{n}}\left(\nu_{1}^{n_{1}} \times \cdots \times \nu_{k}^{n_{k}}\right)\left(d s_{1}, \ldots, d s_{n}\right)
$$

и для каждого $n_{1}, \ldots, n_{k}$ в әтой сумме $\left(B_{1}^{\prime}, \ldots, B_{n}^{\prime}\right)$ равняется

$$
(\overbrace{B_{1}, \ldots, B_{1}}^{n_{1}}, \ldots, \overbrace{B_{j}, \ldots, B_{j}}^{n_{j}}, \ldots, \overbrace{B_{k}, \ldots, B_{k}}^{n_{k}}) .
$$

Для всех $m, n_{1}, \ldots n_{k}$ суммма

$$
\sum_{\pi \in S_{n}} \frac{1}{j_{0} ! \cdots j_{n} !} \gamma_{j_{0}, \ldots, j_{n}}(\pi) A^{j_{n}} B_{\pi(n)}^{\prime} A^{j_{n-1}} \cdots A^{j_{1}} B_{\pi(1)}^{\prime} A^{j_{0}}
$$

в (5.4) может быть заменена на

$$
n_{1} ! \cdots n_{k} ! \sum_{\pi \in \mathscr{P}_{n_{1}, \ldots, n_{k}}} \frac{1}{j_{0} ! \cdots j_{n} !} \gamma_{j_{0}, \ldots, j_{n}}^{\prime}(\pi) A^{j_{n}} B_{\pi(n)}^{\prime} A^{j_{n-1}} \cdots A^{j_{1}} B_{\pi(1)}^{\prime} A^{j_{0}},
$$

əде $\mathscr{P}_{n_{1}, \ldots, n_{k}}$ - множество перестановок $\pi \in S_{n}$, определенных следующей формулой (1.22). Для кажсдого $\pi \in \mathscr{P}_{n_{1}, \ldots, n_{k}}$

$$
\begin{aligned}
\gamma_{j_{0}, \ldots, j_{n}}^{\prime}(\pi)= & \int_{\left(\Delta_{n_{1}} \times \cdots \times \Delta_{n_{k}}\right)(\pi)} \mu\left(\left[0, s_{\pi(1)}\right]\right)^{j_{0}} \mu\left(\left[s_{\pi(1)}, s_{\pi(2)}\right]\right)^{j_{1}} \cdots \mu\left(\left[s_{\pi(n)}, 1\right]\right)^{j_{n}} \\
& \times\left(\nu_{1}^{n_{1}} \times \cdots \times \nu_{k}^{n_{k}}\right)\left(d s_{1}, \ldots, d s_{n}\right)
\end{aligned}
$$

где $\left(\Delta_{n_{1}} \times \cdots \times \Delta_{n_{k}}\right)(\pi)$ - множество, определенное в $(1.22)$.

В следующих двух следствиях так же, как и в предложении 5.5, рассматривается экспоненциальный случай. 
СЛЕДСТВИЕ 5.2. Пусть $k=1,2, \ldots$, и пусть $\mu$ u $\nu_{1}, \ldots, \nu_{k}$ - непрерывные вероятностные меры на борелевской $\sigma$-алгебре $\mathscr{B}[0,1]$. Пусть $A, B_{1}, \ldots, B_{k}$ - әлементы алгебры $\mathscr{L}(X)$. Тогда для всех $t \geqslant 0$ выполнено

$$
\begin{aligned}
& T_{\mu, \nu_{1}, \ldots, \nu_{k}}\left(e^{\left(\tilde{A}+\widetilde{B}_{1}+\cdots+\widetilde{B}_{k}\right) t}\right) \\
& =\sum_{n=0}^{\infty} \frac{t^{n}}{n !} \sum_{n_{1}+\cdots+n_{k}=n}\left[\sum_{\pi \in S_{n}} \int_{\Delta_{n}(\pi)} e^{A t \mu\left(\left[s_{\pi(n)}, 1\right]\right)} B_{\pi(n)}^{\prime} e^{A t \mu\left(\left[s_{\pi(n-1)}, s_{\pi(n)}\right]\right)} \ldots\right. \\
& \left.\quad \times e^{A t \mu\left(\left[s_{\pi(1)}, s_{\pi(2)}\right]\right)} B_{\pi(1)}^{\prime} e^{A t \mu\left(\left[0, s_{\pi(1)}\right]\right)}\left(\nu_{1}^{n_{1}} \times \cdots \times \nu_{k}^{n_{k}}\right)\left(d s_{1}, \ldots, d s_{n}\right)\right]
\end{aligned}
$$

где для каждого $n_{1}, \ldots, n_{k}$ в этой сумме $\left(B_{1}^{\prime}, \ldots, B_{n}^{\prime}\right)$ равняется

$$
(\overbrace{B_{1}, \ldots, B_{1}}^{n_{1}}, \ldots, \overbrace{B_{j}, \ldots, B_{j}}^{n_{j}}, \ldots, \overbrace{B_{k}, \ldots, B_{k}}^{n_{k}})
$$

Для всех $n_{1}, \ldots n_{k}$ сумма $\sum_{\pi \in S_{n}} \int_{\Delta_{n}(\pi)} \ldots$ может быть заменена на

$$
n_{1} ! \cdots n_{k} ! \sum_{\pi \in \mathscr{P}_{n_{1}, \ldots, n_{k}}} \int_{\left(\Delta_{n_{1}} \times \cdots \times \Delta_{n_{k}}\right)(\pi)} \cdots
$$

где $\mathscr{P}_{n_{1}, \ldots, n_{k}} u\left(\Delta_{n_{1}} \times \cdots \times \Delta_{n_{k}}\right)(\pi), \pi \in \mathscr{P}_{n_{1}, \ldots, n_{k}}$, опрелелены в формуле $(1.22) u$ абзаче, следующем за формулой (1.22).

В случае, когда $k=1$ и $\nu=\nu_{1}$, каждый член суммы по перестановкам $\pi \in S_{n}$ в выражении (5.7) равен интегралу

$$
\int_{0}^{1} \int_{0}^{s_{n}} \cdots \int_{0}^{s_{2}} e^{A t \mu\left(\left[s_{n}, 1\right]\right)} B e^{A t \mu\left(\left[s_{n-1}, s_{n}\right]\right)} \cdots e^{A t \mu\left(\left[s_{1}, s_{2}\right]\right)} B e^{A t \mu\left(\left[0, s_{1}\right]\right)} \nu^{n}\left(d s_{1}, \ldots, d s_{n}\right),
$$

где $\nu^{n}\left(d s_{1}, \ldots, d s_{n}\right)=\nu^{n}\left(d s_{\pi(1)}, \ldots, d s_{\pi(n)}\right)$. Таким образом, мы получаем

СЛЕДСТВИЕ 5.3. Пусть $\mu$ и - непрерывные вероятностные меры на борелевской $\sigma$-алгебре $\mathscr{B}[0,1]$. Пусть $A$ и $B$ - әлементы из $\mathscr{L}(X)$. Тогда для всех $t \geqslant 0$

$$
\begin{aligned}
\mathscr{T}_{\mu, \nu}\left(e^{(\tilde{A}+\widetilde{B}) t}\right)=\sum_{n=0}^{\infty} \frac{t^{n}}{n !}\left[\sum_{\pi \in S_{n}} \int_{\Delta_{n}(\pi)} e^{A t \mu\left(\left[s_{\pi(n)}, 1\right]\right)} B e^{A t \mu\left(\left[s_{\pi(n-1)}, s_{\pi(n)}\right]\right)} \ldots\right. \\
\left.\times e^{A t \mu\left(\left[s_{\pi(1)}, s_{\pi(2)}\right]\right)} B e^{A t \mu\left(\left[0, s_{\pi(1)}\right]\right)} \nu^{n}\left(d s_{1}, \ldots, d s_{n}\right)\right]
\end{aligned}
$$

Следующая лемма используется при доказательстве предложения 5.5 и, кроме того, имеет самостоятельный интерес. 
Лемма 5.4. Пусть $A_{1}, \ldots, A_{n}$ принадлежсат $\mathscr{L}(X)$. Пусть $\mu$ - непрерывная вероятностная мера на $\mathscr{B}[0,1]$. Тогда для всех скаляров $\xi_{1}, \ldots, \xi_{n}$ и всех неотрииательных иелых т справедливо равенство

$$
\mathscr{T}_{\mu, \ldots, \mu}\left(\sum_{j=1}^{n} \xi_{j} \widetilde{A}_{j}\right)^{m}=\left(\sum_{j=1}^{n} \xi_{j} A_{j}\right)^{m}
$$

Если $g(z)$ - аналитическая функиия со степенным рядом $\sum_{m=0}^{\infty} c_{m} z^{m}$, равномерно сходящимся на замкнутом диске радиуса $\sum_{j=1}^{n}\left|\xi_{j}\right|\left\|A_{j}\right\|$, то функиия $f:\left(z_{1}, \ldots, z_{n}\right)$ $\rightarrow g\left(\sum_{j=1}^{n} \xi_{j} z_{j}\right)$ принадлежит $\mathbb{A}\left(\left\|A_{1}\right\|, \ldots,\left\|A_{n}\right\|\right)$ и выполнено равенство

$$
f_{\mu, \ldots, \mu}\left(A_{1}, \ldots, A_{n}\right)=g\left(\sum_{j=1}^{n} \xi_{j} A_{j}\right)=\sum_{m=0}^{\infty} c_{m}\left(\sum_{j=1}^{n} \xi_{j} A_{j}\right)^{m}
$$

ДокАЗАтЕЛЬСтво. Для каждого $k=1,2, \ldots$ вероятностная мера $\mu^{k}$ инвариантна относительно перестановок переменных из $[0,1]^{k}$, поэтому для любых перестановок $\pi \in S_{k}$ мера подмножества $\left[0<s_{\pi(1)}<\cdots<s_{\pi(k)}<1\right]$ в $[0,1]^{k}$ равна $1 / k !$.

Из формулы (1.20) следует, что для всех неотрищательных целых $m_{1}, \ldots, m_{n}$ имеет место соотношение

$$
\mathscr{T}_{\mu, \ldots, \mu} P^{m_{1}, \ldots, m_{n}}\left(\widetilde{A}_{1}, \ldots, \widetilde{A}_{n}\right)=\frac{m_{1} ! \cdots m_{n} !}{m !} \sum_{\sigma} A_{\sigma(1)} \cdots A_{\sigma(n)} .
$$

Сумма берется по всем отображениям $\sigma$ множества $\{1, \ldots, m\}$ в $\{1, \ldots, n\}$, которыепринимают значение $j$ в точности $m_{j}$ раз для каждого $j=1, \ldots, n$. Здесь $m=m_{1}+\cdots+m_{n}$. Другими словами, все возможные упорядочения произведения операторов имеют одинаковьй вес. Это является характеристикой разложения правой части (5.9), поэтому (5.9) следует из разложения обеих частей уравнения и применения формулы (5.10).

Вторая часть утверждения следует из того факта, что $\mathscr{T}_{\mu, \ldots, \mu}$ является ограниченным линейным оператором.

Следуюшее утверждение дает разложение типа ряда Дайсона для $e^{(A+B) t}$ относительно непрерьвной вероятностной меры $\mu$. В случае, если $\mu$ является мерой Лебега $\ell$ на $[0,1]$, этот результат хорошо известен [1], [5], [12].

ПРЕДЛОЖЕНИЕ 5.5. Пусть $\mu$ - непрерывная вероятностная мера на борелевской б-алгебре $\mathscr{B}[0,1]$. Пусть $A$ и $B$ - әлементы алгебры $\mathscr{L}(X)$. Тогда для всех $t \geqslant 0$

$$
\begin{aligned}
& \mathscr{T}_{\mu, \mu}\left(e^{(\tilde{A}+\widetilde{B}) t}\right)=e^{(A+B) t}=\sum_{n=0}^{\infty} t^{n}\left[\int_{0}^{1} \int_{0}^{s_{n}} \cdots \int_{0}^{s_{2}} e^{A t \mu\left(\left[s_{n}, 1\right]\right)} B e^{A t \mu\left(\left[s_{n-1}, s_{n}\right]\right)} \ldots\right. \\
&\left.\times e^{A t \mu\left(\left[s_{1}, s_{2}\right]\right)} B e^{A t \mu\left(\left[0, s_{1}\right]\right)} \mu^{n}\left(d s_{1}, \ldots, d s_{n}\right)\right]
\end{aligned}
$$


ДокАЗАТЕЛЬСТво. Первое равенство в (5.11) следует из леммы 5.4. Второе следует из уравнения (5.8) после того, как мы заметим, что для любого $n=1,2 \ldots$ каждый член суммы по перестановкам $\pi \in S_{n}$ равен интегралу

$$
\begin{aligned}
& \int_{0}^{1} \int_{0}^{s_{n}} \cdots \int_{0}^{s_{2}} e^{A t \mu\left(\left[s_{n}, 1\right]\right)} B e^{A t \mu\left(\left[s_{n-1}, s_{n}\right]\right)} \cdots e^{A t \mu\left(\left[s_{1}, s_{2}\right]\right)} B e^{A t \mu\left(\left[0, s_{1}\right]\right)} \mu^{n}\left(d s_{1}, \ldots, d s_{n}\right), \\
& \text { где } \mu^{n}\left(d s_{1}, \ldots, d s_{n}\right)=\mu^{n}\left(d s_{\pi(1)}, \ldots, d s_{\pi(n)}\right) .
\end{aligned}
$$

ЗАмЕчАнИЕ 5.6. Из [13, теоремы $2.4,2.8]$ следует, что в случае, когда $X$ - гильбертово пространство и $A, B$ - ограниченные самосопряженные операторы, действующие на $X$, функциональное исчисление

$$
f \mapsto f_{\mu, \mu}(A, B) \quad \text { для всех } f \in \mathbb{A}(\|A\|,\|B\|)
$$

является сужением на $\mathbb{A}(\|A\|,\|B\|)$ вейлевского исчисления для пары $(A, B)$. Это верно для любой непрерывной вероятностной меры $\mu$ на $[0,1]$, а не только меры Лебега $\ell$. Аналогичные замечания справедливы для самосопряженных элементов $\left(A_{1}, \ldots, A_{n}\right)$ алгебры $\mathscr{L}(X)$.

\section{СПИСОК ЦИТИРОВАННОЙ ЛИТЕРАТУРЫ}

[1] Feynman R. An operator calculus having applications in quantum electrodynamics // Phys. Rev. 1951. V. 84. P. 108-128.

[2] Feynman R. Mathematical formulation of the quantum theory of electromagnetic interaction // Phys. Rev. 1950. V. 80. P. 440-457.

[3] Feynman R., Hibbs A. Quantum Mechanics and Path Integrals. New York: McGraw-Hill, 1965.

[4] Jefferies B., Johnson G. W. Feynman's operational calculi for noncommuting operators: Definitions and elementary properties // Russ. J. Math. Phys. 2001. V. 8. № 2. P. 153-178.

[5] Johnson G. W., Lapidus M. L. The Feynman Integral and Feynman's Operational Calculus. Oxford Math. Monograph. Oxford: Oxford Univ. Press, 2000.

[6] Маслов В. П. Операторные методы. М.: Наука, 1973.

[7] Nazaikinskii V.E., Shatalov V.E., Sternin B.Yu. Methods of Noncommutative Analysis. Studies in Math. V. 22. Berlin-New York: Walter de Gruyter, 1996.

[8] Jefferies B., Johnson G. W., Nielsen L. Feynman's operational calculi for time dependent noncommuting operators (Proc. of Seoul Conference "Feynman Integral and Applications") // J. Korean Math. Soc. 2001. V. 38 (to appear).

[9] Nielsen L. Thesis. Lincoln: Univ. of Nebraska, 1999.

[10] Johnson G. W., Nielsen L. A stability theorem for Feynman's operational calculus // Conference Proc. Canad. Math. Soc. Conference in Honor of Sergio Albeverio's 60th birthday. V. 29, 2000. P. 351-365.

[11] Jefferies B., Johnson G.W. Feynman's operational calculi for noncommuting systems of operators // Pure Math. Report. PM 98/99. Sydney, Australia: School of Mathematics, UNSW, 1999.

[12] Reed M., Simon B. Methods of Modern Mathematical Physics. V. 1. Orlando: Acad. Press, 1980.

[13] Anderson R. F. V. The Weyl functional calculus // J. Funct. Anal. 1969. V. 4. P. 240-267.

(Б. Джеффрис) Университет Южного Уэльса, Австралия 